\title{
Biomolecular condensation of the microtubule-associated protein tau
}

Tina Ukmar-Godec ${ }^{1,2}$, Susanne Wegmann ${ }^{3, *}$, Markus Zweckstetter, ${ }^{1,2,4,{ }^{*}}$

${ }^{1}$ German Center for Neurodegenerative Diseases (DZNE), Von-Siebold-Str. 3a, 37075

Göttingen, Germany.

${ }^{2}$ Department of Neurology, University Medical Center Göttingen, University of Göttingen, Waldweg 33, 37073 Göttingen, Germany.

${ }^{3}$ German Center for Neurodegenerative Diseases (DZNE), Chariteplatz 1, 10117 Berlin, Germany.

${ }^{4}$ Department for NMR-based Structural Biology, Max Planck Institute for Biophysical Chemistry, Am Faßberg 11, 37077 Göttingen, Germany

*Correspondence should be addressed to: S.W. (Susanne.Wegmann@dzne.de), M.Z. (Markus.Zweckstetter@dzne.de). 


\begin{abstract}
Cells contain multiple compartments dedicated to the regulation and control of biochemical reactions. Cellular compartments that are not surrounded by membranes can rapidly form and dissolve in response to changes in the cellular environment. The physicochemical processes that underlie the formation of non-membrane-bound compartments in vivo are connected to liquid-liquid phase separation of proteins and nucleic acids in vitro. Recent evidence suggests that the protein tau, which plays an important role in Alzheimer's disease and other neurodegenerative disorders, phase separates in solution, forms tau phases with microtubules, and associates with phase-separated RNA-binding protein granules in cells. Here we review the experimental evidence that supports the ability of tau to phase separate in solution and form biomolecular condensates in cells. As for other disease-relevant proteins, the physiological and pathological functions of tau are tightly connected - through loss of normal function or gain of toxic function - and we therefore discuss how tau phase separation plays a role for both, and with respect to different cellular functions of tau.
\end{abstract}

Keywords: liquid-liquid phase separation, tau protein, post-translational modifications, neurodegeneration, stress granule

\title{
1. Introduction
}

Cells contain a variety of compartments, which enable the dynamic spatiotemporal coordination of biochemical reactions and processes but lack a surrounding membrane. Increasing evidence suggests that these compartments form and dissolve via liquid-liquid phase separation (LLPS; Table 1) of macromolecules in response to cellular signals [1, 2], such as regulated changes in protein/RNA concentration [3, 4], as well as changes in salt concentration [1], pH [5] and/or temperature $[2,6,7]$. Intracellular compartments, which are not surrounded by membranes, are also named biomolecular condensates (Table 1) [1,2]. Aberrant phase separation, which can lead to the transition from liquid-like into more solid-like phases, has been linked to human disease [8-15]. Moreover, recent studies highlighted the important role of post-translational modifications in the regulation of biomolecular condensation [16] and found that protein LLPS is modulated by phosphorylation of tyrosine [17], serine [18, 19], and threonine [20], methylation of arginine residues [13, 21-23], and acetylation of lysine residues [24-26]. The post-translational modifications can generate binding motifs that enable inter-molecular 
interactions, or increase/decrease the local net charge of residues and thus modulate the critical concentration threshold for protein LLPS [27].

We and others recently reported that the microtubule (MT)-associated protein tau, which is found in insoluble protein deposits in the brains of patients with Alzheimer's disease and other neurodegenerative disorders [28-32], undergoes LLPS [12, 33-35]. Phase separation of tau into liquid-like droplets and alternative forms of LLPS-mediated condensates could therefore be an integral part of tau biology and pathology. In the following we describe key properties of LLPS (chapter 2) and review basic properties of the amino acid sequence and structure of tau (chapter 3). In chapter 4, we discuss the experimental evidence that is currently available with respect to LLPS of tau in vitro, while chapter 5 summarizes observations that tau phosphorylation and acetylation modulate tau condensation. In chapter 6 , we then review some cellular tau functions, in which tau protein condensation could have implications for neurobiology. We also discuss how these neuronal tau phases could play a role in neurodegenerative diseases, in many of which tau appears to be a mediator of neurotoxicity.

\section{LLPS of biomolecules}

\subsection{Physical chemistry of LLPS}

LLPS is a phenomenon inherent to the thermodynamics of liquids [7, 36, 37]. Complex fluids gain unique thermodynamic, rheological, and other physical properties through liquid-liquid demixing: the two states - homogeneous and phase-separated state - have different free energies depending on temperature and pressure [38]. From a thermodynamic point of view, LLPS of macromolecules results from the interplay of the entropy of mixing, which favors a singlephase mixed state, some form of sufficiently strong attractive interactions between molecules favoring a phase-separated state, and the configurational entropy of individual chain molecules, which effectively lowers the entropic penalty of de-mixing as a result of the connectivity of individual polymeric chains (Fig. 1) [2, 7, 27].

The phase behavior can most easily be understood in terms of Flory-Huggins theory of polymer solutions $[39,40]$. According to this theory, the solvent (denoted here by subscript s) and polymer molecules (denoted by subscript $\mathrm{p}$ ), which are composed of $n_{s}$ and $n_{p}$ monomers, respectively, occupy sites on a lattice with a total of $N$ sites. The free energy of the system is then composed of an effective interaction energy between all species (i.e. solvent plus solute/macromolecular species) and the entropy of mixing, $\Delta \mathrm{S}_{\mathrm{mix}}$, of all components (see also Fig. 1). When the system is composed of $m_{s}$ solvent and $m_{p}$ polymer molecules, volume 
fractions $\varphi_{\mathrm{s}}=m_{s} n_{s} /\left(m_{s} n_{s}+m_{p} n_{p}\right)$ and $\varphi_{\mathrm{p}}=m_{p} n_{p} /\left(m_{s} n_{s}+m_{p} n_{p}\right)$, respectively, with $m_{s} n_{s}+m_{p} n_{p}=N$, are obtained. For simplicity, we will assume here a simple solvent and take $n_{s}=1$ and set $n_{p}=n$. Central to the theory are the following assumptions: (i) the number of available configurations of the polymer is approximately equal in vacuum and in solution such that the main contributions to the mixing entropy are translational (i.e. center of mass) degrees of freedom, (ii) local variations in the concentration of monomers are not significantly different from their average concentration, and (iii) all interactions are weak enough to not affect the placement of the chain and solute particles on the lattice sites, respectively. In other words, the entropy is that of a mixture of an ideal gas (solvent plus polymer) and the interaction energy corresponds to the average interaction per pair of particles.

The Flory-Huggins free energy of mixing per lattice site, $\Delta \mathrm{F}_{\mathrm{mix}}$, is a result of the mixing entropy $\Delta \mathrm{S}_{\text {mix }}$ and the effective mixing energy $\Delta \mathrm{E}_{\text {mix }}$; it is expressed in units of thermal energy $\mathrm{k}_{\mathrm{B}} \mathrm{T}$, reads $\Delta \mathrm{f}_{\text {mix }} \equiv \Delta \mathrm{F}_{\text {mix }} /\left(\mathrm{N} * \mathrm{k}_{\mathrm{B}} * \mathrm{~T}\right)=\Delta \mathrm{E}_{\text {mix }}-\mathrm{T} * \Delta \mathrm{S}_{\text {mix }}=\chi * \varphi_{\mathrm{s}} * \varphi_{\mathrm{p}}+\varphi_{\mathrm{s}} * \ln \left(\varphi_{\mathrm{s}}\right)+\varphi_{\mathrm{p}} / \mathrm{n} * \ln \left(\varphi_{\mathrm{p}}\right)$ with $\varphi_{\mathrm{s}}=1$ $-\varphi_{p}$. The mixing entropy always favors a mixed single-phase state, because the number of distinct microscopic configurations is larger in the mixed state. Conversely, the effective interaction energy of mixing, $\Delta \mathrm{E}_{\mathrm{mix}}=\chi * \varphi_{\mathrm{s}} * \varphi_{\mathrm{p}}$, is proportional to the respective volume fractions of the interacting components $\varphi_{\mathrm{s}}$ and $\varphi_{\mathrm{p}}$, with the proportionality parameter $\chi=\mathrm{Z} *\left[\mathrm{E}_{\mathrm{ps}}-\left(\mathrm{E}_{\mathrm{pp}}+\right.\right.$ $\left.\mathrm{E}_{\mathrm{ss}}\right) / 2 \mathrm{~J} /\left(\mathrm{k}_{\mathrm{B}} * \mathrm{~T}\right)$ reflecting the effective mixing energy as a result of destroying z polymer-polymer and solvent-solvent contacts with average energies $E_{p p}$ and $E_{s s}$ respectively, and forming $z$ polymer-solvent contacts with energy $\mathrm{E}_{\mathrm{ps}}$. In situations where $\chi>0$, interactions oppose mixing (i.e. favor demixing/phase separation). In contrast, when $\chi$ is sufficiently positive to favor demixing, $\Delta \mathrm{f}_{\text {mix }}$ has a bi-modal shape and the free energy of the phase-separated state is lower for certain volume fractions (see Fig. 1D). For polyelectrolytes, Overbeek and Voorn extended the theory by including long-range electrostatic interactions within a Debye-Hückel approximation adding an additional energetic term to $\Delta \mathrm{f}_{\text {mix }}$ that is proportional to $\alpha * \varphi_{\mathrm{p}}{ }^{3 / 2}$, where $\alpha$ reflects the strength of electrostatic interactions [41]. Notably, as a result of polymer configurational degrees of freedom the mixing entropy penalty for phase separation is smaller for polymers than for simple solutes.

Because the proportionality parameter $\chi$ depends inversely on temperature, a temperature - the so-called upper critical solution temperature UCST - exists according to Flory-Huggins theory, above which the mixing entropy will dominate and always lead to a mixed state. Polymer systems, however, can also exhibit a lower critical solution temperature (LCST), or more complex phase behavior. LCST results from entropic contributions, which are neglected in the Flory-Huggins theory. These effects include (i) strong polar interactions and 
differences in molecular packing, which induce differences in the thermal expansion coefficient between the components and their mixture [42], (ii) the presence of directionally-specific interactions [43], and (iii) hydrophobic interactions, which are dominated by an entropic contribution that increases with temperature and leads to the presence of terms in $\chi$ that are constant or have a logarithmic dependence on T [44].

\subsection{LLPS of proteins and nucleic acids}

Increasing experimental evidence links LLPS of proteins and nucleic acids to the formation of biomolecular condensates $[1,7]$. Biomolecular condensation is driven by transient multivalent interactions between proteins as well as RNA and DNA [2, 15, 27, 45-47]. Proteins that can undergo LLPS often contain disordered, low-complexity regions [2, 27, 45]. A variety of RNAbinding proteins display modular architectures, in which sequences of disordered regions are appended to RNA-binding domains such as RNA-recognition motif (RRM) domains, RGG repeats, and enzymatic domains [2]. Moreover, homotypic interactions between intrinsically disordered regions alone can drive phase separation, and negatively-charged RNA can further enhance LLPS through interactions with RNA-binding domains and/or disordered protein regions [2].

Coacervation (Table 1) of oppositely charged molecules (e.g. protein and RNA, or protein and polyelectrolyte) promotes LLPS [37, 48], highlighting the importance of electrostatic interactions for biomolecular LLPS [18, 47, 49]. In addition, hydrophobic interactions can influence protein LLPS (please see 2.1. Physical chemistry of LLPS): whereas some condensates dissolve upon increasing temperature with an associated UCST (such as the protein Fused in Sarcoma (FUS)), hydrophobic interactions favor phase separation upon increasing temperature. Indeed, LCST behavior has been observed in vitro for protein and model polypeptide systems [50-53]. Many LLPS proteins belong to the group of RNA-binding proteins. In FUS for example, cation- $\pi$ interactions between tyrosines in its prion-like domain and arginines in the RNA-binding domain are important to facilitate LLPS. Other residues such as glycine, glutamine and serine further contribute to LLPS through modulation of droplet fluidity and hardening [15].

In many in vitro studies focusing on protein LLPS, non-polar agents such as polyethylene glycol (PEG), Ficoll, or dextran are used, in order to mimic conditions of intracellular molecular crowding with estimated biomolecular concentrations of $\sim 100-400$ $\mathrm{mg} / \mathrm{ml}$ occupying $5-40 \%$ of the cytosolic space [54]. In the presence of such crowding agents, the critical protein concentration necessary to undergo LLPS can be markedly lowered. The 
mechanism by which the addition of neutral polymers promotes the condensation of proteins is attributed to excluded volume effects: according to Flory-Huggins theory [39, 55], neutral polymers, which do not interact with themselves or other molecules in the solution, can be described as random coil polymer chains that occupy a certain (crowder concentration and size dependent) space of the solution, which in consequence is not available for other molecules; other molecules become restricted to a certain volume fraction, which increases their concentration in the available volume above a critical concentration for LLPS. Another theoretical description of molecules in a crowded environment, the scaled-particle theory [56], creates excluded volume effects by assuming the crowder molecules as hard spheres with a radius depending on the crowder molecular weight. It has been suggested that crowding may directly impact the conformation of folded proteins [57, 58] and the compactness of intrinsically disordered proteins [59], which might influence their propensity for LPPS. While the addition of non-polar agents accounts for general molecular crowding effects, it neglects the fact that biomolecules present in the cellular environment mostly have a charged surface to be soluble in the aqueous cytosol. Charged molecules, however, can undergo a plethora of specific and unspecific "soft" interactions in a crowded environment $[60,61]$ and in principle can deplete molecules from the pool available for LLPS.

\section{The microtubule-associated protein tau}

\subsection{Amino acid sequence and structure of tau}

The gene encoding the human tau protein, is located on chromosome 17. Alternative splicing of exons 2, 3, and 10 produces six different isoforms of tau in the human central nervous system (CNS) (Uniprot ID 10636; Fig. 2A) [62]. The six isoforms differ in the number of N-terminal inserts $(\mathrm{N} 1, \mathrm{~N} 2)$ and have either three or four $\sim 31$-residue-long, imperfect repeats (R1-R4) in their C-terminal half [63]. The shortest isoform contains 352 residues, the longest isoform in the adult human brain, which is called 2N4R tau or htau40, has 441 amino acids [62, 64]. The imperfect repeats together with two N-terminal proline-rich regions are important for tubulin polymerization and interaction with microtubules (MTs) [65-71].

The amino acid sequence of tau is mostly hydrophilic, contains only a small number of hydrophobic residues, is of low complexity and locally repetitive [64] (Fig. 2B). Tau does therefore not fold into a stable structure [72] but rather fluctuates between many different conformations when not bound to a partner protein [67]. Because of these properties, tau 
belongs to the class of intrinsically disordered proteins [73] and is best studied by Nuclear Magnetic Resonance (NMR) spectroscopy [74] and single-molecule FRET spectroscopy [7579]. The low hydrophobicity of the tau sequence also makes the protein highly soluble [72]. Within the repeat region, however, two hydrophobic hexapeptide sequences are located that are important for aggregation of tau (Fig. 2B) [80]. In addition, the oxidation state of the two native cysteine residues (Cys291 and Cys322; Fig. 2B) influences tau aggregation [81, 82].

When the tau protein oligomerizes and aggregates into amyloid fibrils it acquires $\beta$ structure [83-87]. Cryo-electron microscopy of paired and straight helical filaments isolated from the brain of a patient with Alzheimer's disease revealed the molecular details of the cross$\beta$ structure within the core of insoluble tau deposits $[88,89]$. Outside of this rigid core, tau remains flexible. This allows the $\mathrm{N}$-terminus to contact the hexapeptides in the core structure and thus establish an epitope that is recognized by the conformation-specific antibodies Alz50 and MC-1 [90-92]. Aggregated tau is unable to bind to MTs, which in turn affects their dynamic instability [93]. The level of aggregation of tau into neurofibrillary tangles correlates with the degree of cognitive decline in Alzheimer's disease [94, 95]. Mutations in the protein sequence modulate tau's ability to form tangles and lead to frontotemporal dementia (FTD) $[96,97]$.

Because of a small number of hydrophobic residues and the low complexity of its amino acid sequence (Fig. 2B), the tau protein displays very little tendency to aggregate [98]. Negatively charged co-factors (e.g. heparin and RNA) or a specific, yet unknown, phosphorylation pattern are thus critical to aggregate tau $[99,100]$. Consistent with the importance of co-factors for pathogenic tau aggregation, neurofibrillary tangles isolated from the brains of patients with Alzheimer's disease contain RNA and glycosaminoglycans [99, 100]. Moreover, proteases and endopeptidases liberate the repeat domain of tau [101, 102], which displays a higher tendency to aggregate than full-length tau [98].

\subsection{Post-translational modification of tau}

In vivo tau is highly post-translationally modified, including phosphorylation, glycation, acetylation and glycosylation $[103,104]$. Post-translational modifications of tau modulate tau structure [105-108], tau/MT-interaction [106, 108], tau/actin-interaction [109], tau localization [110], deposition and neurotoxicity [111]. For detailed reviews of post-translational modifications of tau we refer to $[104,112]$.

Phosphorylation is the most common post-translational modification of tau [103, 104]. It was suggested that increased tau phosphorylation decreases its affinity for MTs and promotes 
tau self-aggregation, resulting in destabilization of the neuronal cytoskeleton [103, 104]. Tau phosphorylation is regulated by a large number of kinases and phosphatases with the degree of tau phosphorylation determined by a fine balance of their activity. A perturbation of this balance will result in abnormal phosphorylation of tau in Alzheimer's disease.

Lysine acetylation in tau is elevated during cellular stress [113]. By blocking tau degradation, lysine acetylation dysregulates tau homeostasis [113], impedes tau binding to MTs [114] and promotes synaptic dysfunction [115].

Twelve sites of glycation were detected on tau protein [116]. Besides the production of free radicals, glycation might contribute to a blockade of tau degradation and thereby promote its pathological accumulation $[117,118]$. Glycation promotes tau polymerization and stabilizes aggregated tau protein, but does not by itself induce tau aggregation [119].

Glycosylation is the covalent attachment of oligosaccharides to a protein. During Nglycosylation a sugar is attached to the amine radical of asparagine residues, while Oglycosylation involves sugar attachment to the hydroxyl radical of serine or threonine residues. Tau proteins extracted from brains of Alzheimer's disease patients are abnormally glycosylated $[120,121]$. In vitro, deglycosylation of tau tangles converts tangles back into bundles of straight filaments and restores their accessibility to MTs [120, 121]. Tau glycosylation also decreases tau phosphorylation [122-124], potentially as a result of a competition between phosphorylation and glycosylation for the same sites. Through such competitive mechanisms, O-glycosylation could protect tau from disease-associated hyperphosphorylation [103, 104].

\section{Tau LLPS}

In the following, we review the experimental results describing LLPS of the protein tau (Fig. 3A). We start with self-coacervation, which is spontaneous LLPS in the absence or presence of non-polar crowding agents (Table 1). Subsequently, we discuss LLPS promoted by negatively charged co-factors, in particular RNA (complex coacervation; Table 1). Finally, we review the evidence that post-translational modifications influence phase separation of tau in vitro.

\subsection{Self-coacervation of tau}

Bioinformatic analysis suggests that the amino acid sequence of tau encodes a high propensity for granule formation (Fig. 3B) [33]. Consistent with this prediction some of us showed showed that the K18 polypeptide, which comprises residues 244-372 of 2N4R tau (Fig. 2), can phase 
separate in vitro into liquid-like droplets in the absence of molecular crowding agents [33]. Self-coacervation of K18 required high protein concentration $(>50-100 \mu \mathrm{M})$, was promoted when the $\mathrm{pH}$ of the solution approached the protein's $\mathrm{pI}$, and reached a maximum at about 37 $45{ }^{\circ} \mathrm{C}$ [33]. At temperatures below $\sim 15{ }^{\circ} \mathrm{C}$, LLPS of $\mathrm{K} 18$ did not occur, in agreement with LCST-like phase behavior. NMR spectroscopy showed that the KXGS motifs, which can be phosphorylated by the MT-associated protein/MT affinity-regulating kinase 2 (MARK2) [106, 125], are hot spots in the process of self-coacervation of K18 [126]. Within these droplets, K18 remained highly flexible, but experienced small changes in secondary structure towards $\beta$ hairpin-like structure [126]. In addition, interactions between the four pseudo-repeats of K18 were strongly enhanced in the phase separated state [33]. These interactions could occur either within a single K18 molecule or between different K18 molecules, and are likely to originate from the increase in K18 concentration in the interior of droplets [33]. Notably, tau has an unusually high solubility ( $>200 \mu \mathrm{M})$ and a high diffusivity [127], therefore its condensation is expected to be to a large extent governed by entropic effect.

Because of the strong increase in protein concentration in the interior of condensed phases, liquid droplets might provide a favorable environment for protein aggregation $[1,2,7]$. Consistent with this hypothesis, we found that addition of heparin to preformed K18 droplets changed the morphology of K18 droplets and resulted in amyloid fibrils [33]. This observation was in agreement with many studies showing that efficient aggregation of tau into amyloid fibrils is only achieved in the presence of negatively charged co-factors, such as heparin, RNA or acidic peptides (e.g. the C-terminal tails of tubulin) [99, 100, 128-130]. The importance of LLPS as an initial step for pathogenic misfolding and aggregation of K18 was further supported by the observation that K18 LLPS in the absence of heparin and K18 fibrillization in the presence of heparin showed a similar dependence on temperature, ionic strength, and $\mathrm{pH}$ [33]. Notably, studies with a shorter tau fragment (K19; residues 244-372 of 2N4R tau but without residues 275-305, i.e. repeat R2; Fig. 2) suggested that each imperfect repeat of tau acts as a single interaction motif, such that the total number of repeats influences the multivalency of tau self-coacervation [33].

Independently, Hernández-Vega and coworkers showed that 2N4R tau (htau40; see Fig. 2A) tagged with enhanced green fluorescent protein (EGFP) undergoes LLPS in presence of molecular crowding agents [35]. htau40-EGFP droplet formation was influenced by the size and concentration of the crowding agent, and was promoted by larger and more concentrated crowders. In addition, LLPS of tau depends on ionic strength: low (high) salt concentrations promote (inhibit) LLPS of K18 and htau40-EGFP, suggesting that electrostatic interactions are 
important for LLPS of tau $[33,35]$. Hernández-Vega et al. also found that tubulin molecules are recruited into htau40-EGFP droplets, with the concentration of tubulin inside the droplets being $\sim 10$-fold higher than the concentration outside [35]. In turn, tubulin also stimulated the formation of htau40-EGFP droplets, suggesting a positive feedback mechanism between tubulin enrichment and LLPS of tau. Even at overall concentrations of tubulin that were four times lower than the concentration needed for spontaneous nucleation of MTs in vitro, MT bundles nucleated in htau40-EGFP droplets. Growth of MT bundles deformed htau40-EGFP droplets into rod-like shapes. Further analysis showed that tubulin polymerization within htau40-EGFP droplets is promoted by a combination of local tubulin concentration and spatial confinement to the 'container' of the htau40-EGFP droplet. Notably, the addition of heparin disrupted the interaction of htau40-EGFP with MT bundles and lead to the re-formation of spherical htau40-EGFP droplets and naked MT bundles. These observations suggest that tau droplets stabilize MT bundles by keeping the tubulin concentration in the droplet above a critical value and/or by directly stabilizing the MT lattice [35].

In a further independent study, Wegmann et al. showed that both wild-type htau40 tagged with green fluorescent protein (GFP) and htau40-GFP carrying mutations found in frontotemporal dementia (FTD) can undergo phase separation in vitro and form condensates in living neuronal cells [12]. Condensation of htau40-GFP occured in the cytosol of cells with a sufficiently high expression level of tau. Some droplet-like accumulations of htau40-GFP in axons moved retro- as well as anterogradely. In addition, the accumulations of htau40-GFP were less mobile than those formed by the N-terminal projection domain (residues 1-256 of 2N4R tau), potentially due to the inability of the latter to bind MTs via the repeat domain. Fluorescence recovery after photobleaching profiles of the observed htau40-GFP accumulations were consistent with highly mobile tau species. htau40-GFP droplets did not colocalize with membrane-bound organelles, such as lysosomes, endosomes or the endoplasmic reticulum. Mutations of tau, which are associated with FTD and promote the pathological aggregation and deposition of tau [131], induced LLPS of htau40-GFP at lower protein concentrations when compared to the wild-type protein, strengthening the connection between LLPS and pathogenic aggregation of tau [12, 33, 35].

\subsection{Complex coacervation of tau with RNA}

Intracellular non-membrane-bound organelles, such as processing bodies and stress granules, contain a large number of RNA and proteins, including tau [19, 45, 133-137]. The groups of 
Han and Kosik showed that tau binds RNA in two stages that are mediated, respectively, by (i) strong binding with nanomolar dissociation constants at a tau:RNA molar ratio of 2:1, and (ii) weak association between tau multimers and RNA to form higher order complexes. Interaction of the tau construct $\Delta$ tau187 (residues $255-441$ of htau40), and in a less robust manner also htau40, resulted in the formation of liquid-like droplets [34]. Similar to K18 droplets, which are formed by self-coacervation [33], $\Delta$ tau187 remained highly mobile in $\Delta$ tau187/RNA droplets. The formation and stability of $\Delta$ tau187/RNA droplets was reversible and sensitive to increased ionic strength, $\Delta$ tau187:RNA molar ratio, and temperature. Notably, binding of tau to tRNA was found to be stronger when compared to the interaction with other RNA species, despite tau lacking a known RNA-binding motif [34].

In a follow up study, the groups of Han and Kosik showed that, not only selfcoacervation of K18 displays LCST behavior (as shown by the Zweckstetter/Mandelkow labs [33]), but also $\Delta$ tau187/RNA coacervates [138]. $\Delta$ tau187/RNA coacervation was reported to occur in a narrow range of temperature and protein concentration. While $\Delta \operatorname{tau} 187$ maintained a state of dynamic flexibility in the coacervates, addition of heparin drove the system into a fibrillar state, in agreement with observations made for tau droplets formed by self-coacervation $[12,33]$. Computer simulations further suggested that the tau/RNA ratio in cellular physiological conditions is located near a binodal phase boundary, implying that small and subtle changes in the cellular environment might be sufficient to induce tau condensation. The authors of [138] further suggested that biological mechanisms that increase the concentration of tau or RNA (for example osmotic stress), as well as $\mathrm{pH}$, could drive complex coacervation of tau/RNA in neurons.

Recently, some of us investigated the ability of the tau constructs K18, K25 (residues 1-243 of 2N4R tau but without residues 55-73, i.e. N1, N2 inserts; Fig. 2) and htau40 to undergo complex coacervation with RNA [26]. All three tau proteins underwent LLPS but required different amounts of RNA. K18 with the highest pI required the largest amount of RNA to undergo phase separation, while htau40 with the lowest $\mathrm{pI}$ required the smallest amount of RNA, highlighting the importance of electrostatic interactions in complex coacervation of tau. In addition, we found that htau40 phase separated at $\mathrm{pH} 7.4$ in presence of the molecular crowding agent dextran, most likely because of the ampholytic nature of htau40.

\section{Modulation of tau condensation by post-translational modifications}




\subsection{Modulation of tau LLPS by phosphorylation}

Intrinsically disordered proteins such as tau are regulated by a variety of post-translational modifications [103, 139, 140]. In the brain of patients with Alzheimer's disease, tau is hyperphosphorylated [141-144]. A large number of kinases can phosphorylate serine and threonine residues of tau in vitro $[105,145]$. A kinase, which phosphorylates serine residues in the repeat domain of tau [106], is the MT-associated protein/MT affinity-regulating kinase 2 (MARK2) [125]. Phosphorylation of tau by MARK2 influences both tau aggregation and the binding of tau to MTs [146]. Consistent with the importance of the repeat region of tau for condensation, MARK2-phosphorylation of this region lowered the critical concentration for K18 LLPS $[12,33]$. In addition, non-phosphorylated K18 was recruited into preformed droplets of MARK2-phosphorylated K18 [33], suggesting that condensates formed by phosphorylated tau could promote condensation of different tau species.

The importance of tau phosphorylation for LLPS was further supported by the finding that a full-length tau protein (P-tau), which was recombinantly produced in Sf9 insect cells and had a phosphorylation state similar to tau extracted from Alzheimer's disease brains [147], rapidly formed droplets in solution at near physiological concentrations $(\sim 1-2 \mu \mathrm{M})$ upon addition of molecular crowding agents (Fig. 4) [12]. LLPS of P-tau in the presence of molecular crowding agents was furthermore found to be insensitive to increasing ionic strength $(\leq 1 \mathrm{M}$ $\mathrm{NaCl}$ ), suggesting that screening of electrostatic interactions had a smaller effect on LLPS of phosphorylated when compared to non-phosphorylated tau. In addition, LLPS of P-tau was robust against changes in $\mathrm{pH}$, as well as high concentrations $(\leq 1 \mathrm{M})$ of mono- $(\mathrm{KCl})$ or bivalent $\left(\mathrm{MgCl}_{2}\right)$ cation salts [12]. Wegmann et al. also observed that the C-terminal half of tau (P-tauCt; residues 242-441 of 2N4R tau), is able to undergo LLPS in a NaCl-insensitive ( $\leq 1 \mathrm{M} \mathrm{NaCl})$ manner [12]. The addition of heparin or RNA to P-tau lead to spontaneous in vitro formation of droplets in the absence of molecular crowders, with extended incubation resulting in the formation of Thioflavine S-positive tau aggregates [12]. Moreover, hyperphosphorylated high molecular weight tau species derived from Alzheimer's disease brain phase separated into liquid-like droplets [12].

\subsection{Tau LLPS and acetylation}

Increasing evidence connects acetylation of tau to tau toxicity and pathology $[148,149]$. In cells, tau can be acetylated by different lysine acetyltransferases (KAT) such as CREB or p300 
[148, 149], and deacetylated by deacetylases including sirtuin 1 [113]. Recent studies highlight the importance of the acetylation state of intrinsically disordered proteins/protein regions for biomolecular condensation [24-26]. Ferreon et al. investigated the influence of p300acetylation on LLPS of htau40 [24]. They found that acetylation disfavors LLPS of htau40, inhibits heparin-induced aggregation, and impedes the ability of tau to promote MT assembly. We further showed that tau acetylation by CREB reverses LLPS of htau40 and K18 [26]: liquid droplets of htau40 formed in presence of dextran, as well as of K18 formed in presence of RNA, gradually shrinked and were ultimately dissolved (Fig. 5A, B). NMR spectroscopy of unmodified and acetylated htau40 in presence of increasing RNA concentrations further revealed that acetylation attenuates binding of RNA to tau [26]. Notably, bioinformatic analysis showed that lysine is the most abundant amino acid in disordered regions of proteins found in processing bodies, suggesting that acetylation might influence a wide range of intracellular condensation processes [26].

\section{Roles of tau phases in neuronal biology and neurodegeneration}

\subsection{Interplay of condensed tau phases with tubulin and MTs}

Inhomogeneous coverage of MTs by tau and condensed phases of tau have been connected to its function as a MT-binding protein [150-153]. However, the recently discovered physicochemical state of liquid-like tau phases opens new paths of interpreting old and novel observations. Tau is expressed in neurons of the central nervous system, where its major role is the binding and regulation of axonal MT dynamics. The MT-binding of tau has been characterized in vitro and tau has been shown to regulate the nucleation, growth, and stability of MTs [154, 155].

Recently, it was shown that the recruitment of tubulin into liquid-like droplets of tau is a potent way to initiate the assembly, growth, and bundling of MTs in vitro using recombinant proteins [35]. On assembled MTs, the liquid tau phase forms a sheet-like coating, which might stabilize and protect MTs. Mechanistically, the process is facilitated through interaction of the positively charged tau repeat domain with the negatively charged MT surface, generated by the protrusion of the acidic C-terminal tail of tubulin $[156,157]$. Such "complexation" of anionic and cationic peptides or protein parts resembles aspects of complex coacervation. In fact, the concept that MT-assembly is driven by complex coacervation with cationic co-factors has been introduced early on in research of MTs and MT-associated proteins [158]. Notably, the coating 
of MTs by tau was found to be rather inhomogeneous with tau "patches", or "islands", appearing along the longitudinal axis of MTs in vitro and in cells (Fig. 6) [151, 159, 160].

The size of the tau "patches" on MTs is dynamic and depends on the tau concentration in the surrounding medium. In fact, at low tau concentration $(\sim 0.5-20 \mathrm{nM})$, the coexistence of two distinct tau phases with different physicochemical behavior has been suggested: in condensed tau patches, which form spontaneously and cooperatively on the MT surface at low solution concentrations of tau $[159,160]$, tau has a long dwell time and appears static on the MT surface. In addition, the exchange with tau molecules in the surrounding diffuse phase was steady but slow and tau patches could grow on the edges by integrating tau molecules from the surrounding solution. The other diffuse phase of tau on MTs - outside of the tau patches - is characterized on the other hand by short dwell times of tau, resembling the diffusion of tau molecules on the MT lattice [161, 162]. Structural modifications of the MT lattice, for example by stabilizing MTs with paclitaxel or by altering the acetylation state of tubulin $[150,152]$, influenced tau binding and island formation. In addition, patches of condensed tau were observed preferentially in areas of curved MTs [153, 160]. Binding of tau to less-dense areas of the MT lattice was also supported by a recent study demonstrating the interplay between tau and Map6 in the regulation of labile and stable regions of MTs, which is important in the growth cone and at axon branches [163]. It is well possible that free and/or MT-bound condensed phases of tau play a role in the regulation of local repair, branching, and outgrowth of MTs in these areas. How tau is distributed on MTs in vivo is still unknown, but some indication exists that its distribution in the axon is inhomogeneous as well [164].

In the context of its function as MT-binding protein, it is intuitive that tau also plays a role in axonal transport $[165,166]$. Indeed, it has been shown that "patches" of condensed tau on MTs alter the processivity of different kinesins and dynein in a motor protein specific manner $[151,159,160]$. In addition, the tau patches appear to locally protect MTs against the disassembly by proteins like katanin and spastin $[159,160]$. A deregulation in the equilibrium of MT-related tau condensation processes - for example by aberrant phosphorylation or other post-translational modifications of tau that occur during neurodegenerative diseases - could favor or prevent tau condensation, and thereby cause fatal failure in MT stability, repair, and axonal transport. In fact, all these phenotypes are early signs of neurotoxicity and can be observed prior to neuronal death in tau-related neurodegenerative diseases such as Alzheimer's disease. Furthermore, an aberrantly excessive nucleation of MTs by missorted phosphorylated tau in the neuronal soma could explain the recently reported MT-induced deformation of the nuclear membrane in FTD patient-derived neurons [167]. 


\subsection{Tau association with RNA-binding protein granules}

Local translation of mRNA into protein is especially important in cells, which have a highly asymmetric polarized cell architecture such as neurons: the neuronal cell soma, the dendritic processes and post-synapses, and the axon and pre-synapses each have to provide a different functional micro-environment with different ensembles and concentrations of proteins and other biomolecules. Such spatial distinction is necessary to maintain neuronal function, and mRNA transport into distal areas and local protein expression are essential. mRNA molecules are often transported along MTs in association with RNA-binding proteins (RNPs) granules; in fact, the assembly of RNP granules was the first process for which the coacervation of proteins with RNA molecules was shown to be the driving force [135]. Tau mRNA itself has been shown to colocalize with RNPs [168] and to travel to the distal axon together with the RNP granule protein $\mathrm{HuD}$, where it becomes locally translated into protein $[169,170]$.

In stress conditions, cells utilize LLPS to acutely and reversibly assemble cytoplasmic RNP granules (stress granules, SGs) that contain different RNPs, specific RNAs, and enzymes that are needed for protein translation or mRNA silencing, necessary for the cellular response to the stress [16]. The SG composition appears to vary with the applied stress [171]. In many cases, the formation of cytosolic SGs involves the translocation of otherwise primarily nuclear stress granule proteins. Notably, also small amounts of tau can be found in cytosolic SGs, and the presence of tau in SGs containing the RNP TIA-1 has been shown in different cells including neurons, as well as in the brain of tau transgenic mice and human post-mortem brain from Alzheimer's disease patients [133, 172].

We recently showed that the three recombinantly expressed and purified tau proteins K18, K25 and htau40 colocalize with SGs when added independently to semi-permeabilized HeLa P4 cells, which were stressed by the proteasome inhibitor MG132 [26]. K25 showed the strongest and K18 the weakest colocalization, suggesting that the repeat domain of tau could have an inhibitory effect on SG association. Interestingly, the relative abundance of arginine residues in these three tau proteins decreases in the order $\mathrm{K} 25>\mathrm{htau} 40>\mathrm{K} 18$, hinting towards a potential correlation between relative arginine content and SG colocalization. Additionally, the authors showed that tau acetylation significantly reduces the colocalization of tau with SGs (Fig. 5C). Both results suggested that SGs might also have a potential neuroprotective function. 
The role of tau in RNPs is not known but could be related to its high affinity to RNA and could involve, for example, alterations in stress-response mRNA translation in SGs or the RNA transport by RNP granules. It is also not yet known if the interaction of tau with SGs relies on co-condensation or some other molecular binding mechanism. In the disease context, stress granules seem to play a role in tau-induced neurotoxicity, since the removal of TIA-1 at least on the mRNA level - rescues neuronal death in a transgenic FTD mouse model expressing mutant tau [173]. The removal of TIA-1 increased the amount of tau aggregation as well as the neuronal survival in these mice, suggesting that TIA-1 and tau associated with SGs may mediate tau toxicity in vivo. On the other hand, TIA-1 and other RNPs seem to accumulate with cytosolic phosphorylated-tau deposits in the brain of tau mutant mice [174]. Hence, the interaction of RNPs with pathological tau could change the normal neuronal SG biology in different ways: the association of tau proteins with SGs can alter the normal SG function in stress-response, and RNPs that get sequestered into pathological tau inclusions could lose their normal function. In both cases, the cellular stress response would be impaired. Importantly, there is also an interaction between tau mRNA and RNPs: tau mRNA is found in axonal RNPs of neuronal cells [170], and SG formation in neuronal cells induces a shift in the splicing of tau mRNA and, hence, in protein isoform expression [168]. These findings suggest an important, and yet not understood, aspect of neuronal tau translation and transcription regulation by RNPs and SGs, whose role in neurodegeneration needs more attention. How SGs change the pathophysiology of tau, and how tau changes the physiology of SGs needs to be clarified.

Some RNPs that play a role in normal neuronal stress response are also associated with neurodegenerative diseases, for example the proteins FUS [8], the transactive response DNA binding protein $43 \mathrm{kDa}$ (TDP-43) [9], and heterogeneous nuclear ribonucleoprotein A1 (hnRNPA1) [45]. These proteins also form intracellular protein aggregates in amyotrophic lateral sclerosis (FUS, TDP-43, and hnRNP) and FTD (TDP-43). Disease associated mutations or post-translational modifications can lead to an aberrant condensation and aggregation of LLPS proteins $[9,20,22,175]$. Similarly, tau LLPS is promoted by FTD mutations and pathology-associated phosphorylation $[12,33]$. Until now, however, there is no direct proof that liquid-like condensates of proteins, including tau, which undergo LLPS in vitro or in cells, exist in the living brain. However, at least for tau there is some evidence that droplet-shaped tau accumulations can emerge in the living mouse brain expressing fluorescent protein-tagged human tau and that pathological tau isolated from Alzheimer's disease brains is able to undergo LLPS ex vivo [12]. 


\section{Conclusion}

During the last two years, increasing evidence has been accumulated that the microtubuleassociated protein tau can phase separate in solution, forms tau phases with tubulin and on microtubules, and associates with biomolecular condensates in cells. However, we still know little about the molecular and structural properties of tau in liquid-like droplets and cellular condensates, how these properties depend on post-translational modifications, and how they change in the diseased or ageing brain. To validate the relevance of tau LLPS for human neurodegenerative diseases, it will be important to experimentally close the gap between in vitro liquid-like protein droplets and tau condensation in the brain, in order to gain insights into the contribution of liquid-liquid phase separation and cellular condensation for tau biology and pathology.

\section{Acknowledgments}

We thank Dr. S. Hutten for providing the images of stress granules in HeLa P4 cells. S. W. and M. Z. were supported by the German Science Foundation through SPP2191 (M. Z. through DFG 71/9-1). M. Z. was supported by the advanced grant of the European Research Council (LLPS-NMR, no. 787679)

\section{Competing financial interests}

The authors declare no competing financial interests.

\section{Appendix:}

Table 1. A list of terms used in protein phase separation 
Biomolecular condensates: Intracellular compartments that are not surrounded by a membrane. Examples: stress granules processing bodies, germ granules or cajal bodies.

Membrane-bound organelle (compartment): Cellular compartment that is enclosed by a lipid bilayer membrane. Examples: Golgi apparatus, ribosomes, nucleus or mitochondria.

Phase separation: The transformation of a single-phase system into a multi-phase system that depends on the Gibbs free energy of mixing. For example, two immiscible liquids like water and oil droplets.

Liquid-liquid phase separation: A process in which two liquids demix and form non-miscible phases.

Coacervation: A process in which two phases co-exit, a polymer or colloid-rich phase (coacervate phase or coacervate droplets) and a very dilute phase (liquid). The name "coacervate" derives from the Latin coacervare, meaning "to assemble together or cluster".

Complex coacenvation: Refers to liquid-liquid phase separation that results when solutions of two oppositely charged molecules are mixed.

Self-coacervation: The formation of a coacervate phase without the addition of the second component, e. g. a negatively charged co-factor. It typically involves multivalent or ampholytic macromolecules.

\section{Figures and figure legends}

A

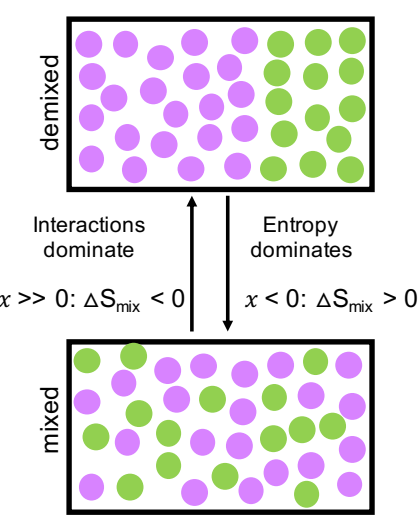

C

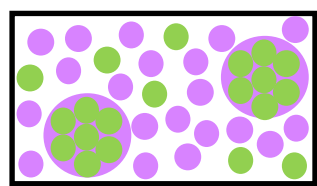

B

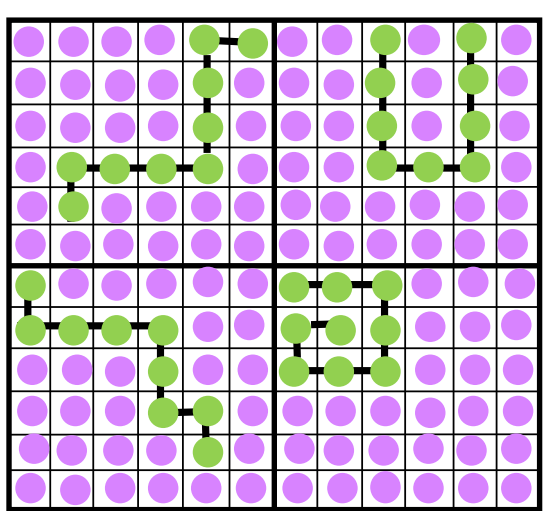

$\mathrm{E}_{\text {protein-protein }(\mathrm{pp})}$

$E_{\text {protein-solvent (ps) }}$

$E_{\text {solvent-solvent(ss) }}$

Demixing (phase separation) can occur if

$E(\bullet \bullet)+E(\bullet \bullet)<2 E(\bullet \bullet)$

E

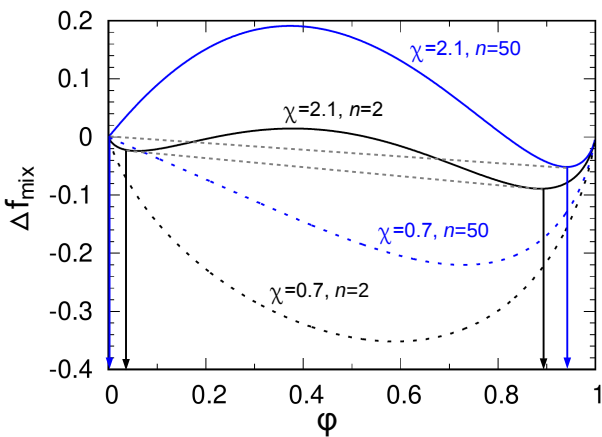

Figure 1. LLPS depends on the interplay between mixing entropy $\left(S_{\text {mix }}\right)$ and effective mixing energy $\left(E_{\text {mix }}\right): \Delta f_{\text {mix }} \equiv \Delta F_{\text {mix }} /\left(N * k_{B} * T\right)=\Delta E_{\text {mix }}-T * \Delta S_{\text {mix }}$ [39-42]. (A) Mixing entropy favors a single-phase mixed state (bottom). Attractive interactions between proteins (green balls) can drive the system into a phaseseparated state (top) when $\chi=\mathrm{Z} *\left[\mathrm{E}_{\mathrm{ps}}-\left(\mathrm{E}_{\mathrm{pp}}+\mathrm{E}_{\mathrm{ss}}\right) / 2\right] /\left(\mathrm{k}_{\mathrm{B}} * \mathrm{~T}\right)>>0$, where $\mathrm{E}_{\mathrm{pp}}, \mathrm{E}_{\mathrm{ss}}$ and $\mathrm{E}_{\mathrm{ps}}$ are interaction 
energies between pairs of proteins, between solvent molecules, and between protein and solvent, respectively [27]. (B) The chain flexibility of polypeptides (here illustrated with $n_{p}=n=9$ ) effectively lowers the entropic penalty associated with phase separation and thus promotes phase separation of polymeric mixtures $[27,39,40]$. (C) In the phase-separated state two liquids exist, which have the same chemical potential but different composition with the droplet phase (green) strongly enriched in protein. (D) Mixing free energy $\Delta \mathrm{f}_{\text {mix }}$ per unit of $\mathrm{k}_{\mathrm{b}} \mathrm{T}$ as a function of the volume fraction of the polymer $\varphi$. When the Flory interaction parameter $\chi$ is sufficiently small (black and blue dashed lines) mixing entropy dominates and leads to a mixed phase, because the free energy of the single-phase system is always smaller than any linear combination of two phases (i.e. a straight line between any two points on the $\Delta \mathrm{f}_{\text {mix }}$ function always lies above). Notably the mixing free energy for longer chains at any fixed $\varphi$ is always smaller, which readily depicts that chain degrees of freedom lower the mixing entropy (see text for more details). As soon as $\chi$ becomes large enough the mixing free energy is not a convex function of volume fraction anymore and hence there exists a region of $\varphi$ where a linear combination of two phases with a composition denoted by the arrows (see dashed grey lines) has a smaller free energy. In these regions of composition, LLPS occurs. (E) Temperature dependence of LLPS. According to FloryHuggins theory, which neglects entropic effects in interactions, the interaction parameter $\chi$ is inversely proportional to temperature. With increasing temperature, interactions thus become less important for phase behavior, and eventually - at the so-called upper critical solution temperature (UCST) - mixing entropy dominates and results in a mixed/non-phase-separated state. In presence of pronounced entropic effects involved in the interactions, including strong polar interactions and differences in molecular packing or in the presence of directionally-specific and hydrophobic interactions, a lower critical solution temperature (LCST) can arise as a result of $\chi$ being constant or having a logarithmic dependence on temperature. 


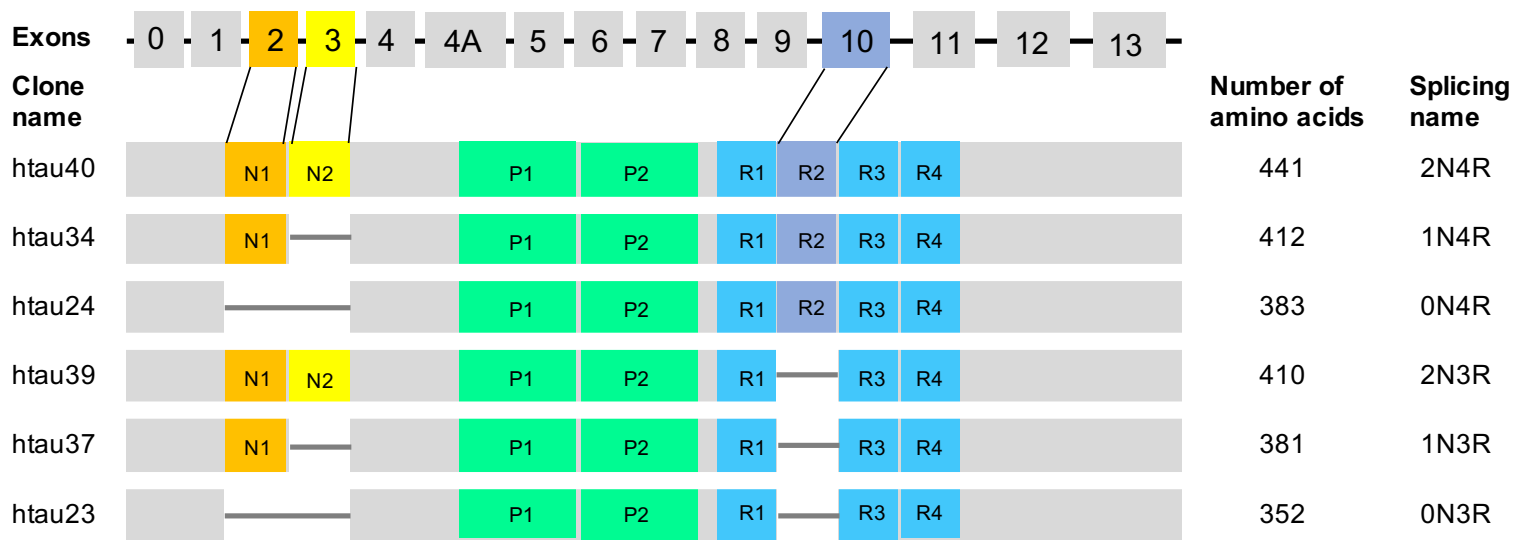

B

htau40 amino acid sequence

MAEPROEFEV MEDHAGTYGL GDRKDQGGYT MHQDQEGDTD AGLKESPLQT $\mathbf{5 0}$ PTEDGSEEPG SETSDAKSTP TAEDVTAPLV DEGAPGKQAA AQPHTEIPEG 100 TTAEEAGIGD TPSLEDEAAG HVTOARMVSK SKDGTGSDDK KAKGADGKTK 150 IATPRGAAPP GQKGQANATR IPAKTPPAPK TPPSSGEPPK SGDRSGYSSP 200 GSPGTPGSRS RTPSLPTPPT REPKKVAVVR TPPKSPSSAK SRLQTAPVPM 250 PDLKNVKSKI GSTENLKHOP GGGK OTINIK KLDLSNVQSK CGSKDNIKHV 300 PGGGS PVDLSKVTS KCGSLGNIHH KPGGGQVEVK SEKLDFKDRV 350 OSKIGSLDNI THVPGGGNKK IETHKLTFRE NAKAKTDHGA EIVYKSPVVS 400 GDTSPRHLSN VSSTGSIDMV DSPOLATLAD EVSASLAKOG L
Domain organization of htau40

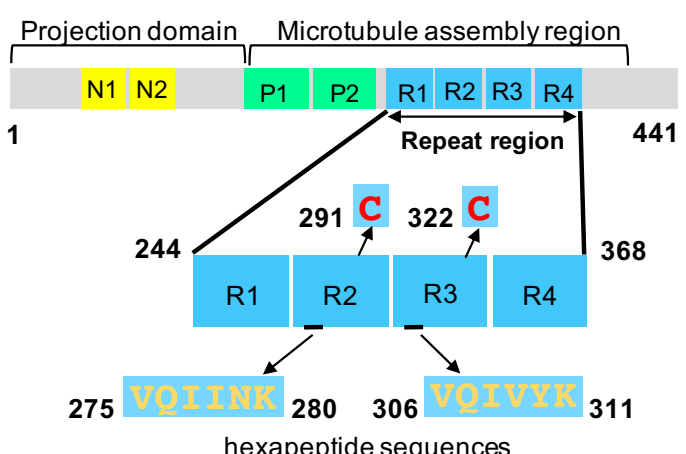

Figure 2. Splicing isoforms, amino acid sequence and domain organization of tau protein. (A) MAPT (microtubule-associated protein tau) gene encodes six tau isoforms in the human brain that result from alternative splicing of exons 2,3 , and 10 . The isoforms differ in the number of N-terminal inserts $(\mathrm{N} 1, \mathrm{~N} 2)$ and in the number of imperfect repeats $(\mathrm{R} 1 / \mathrm{R} 2 / \mathrm{R} 3 / \mathrm{R} 4$ in 4-repeat tau and R1/R3/R4 in 3-repeat tau) and thus in their amino acid sequence. P1 and P2 are two regions of tau that are rich in proline residues. (B) (left) Amino acid sequence of htau40 (2N4R tau). Two hydrophobic hexapeptide sequences are colored in yellow and the two native cysteine residues in red. (right) Domain organization of htau40 indicating the positions of the hexapeptide sequences in R2 and R3, as well as the cysteine residues $\mathrm{C} 291$ and $\mathrm{C} 322$. 
A

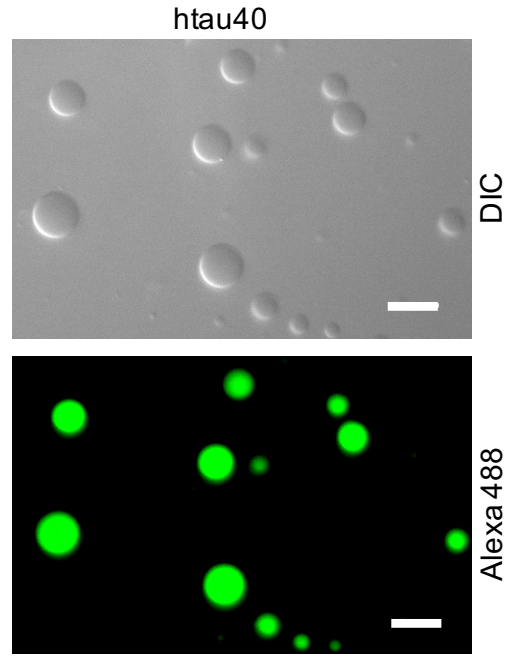

B

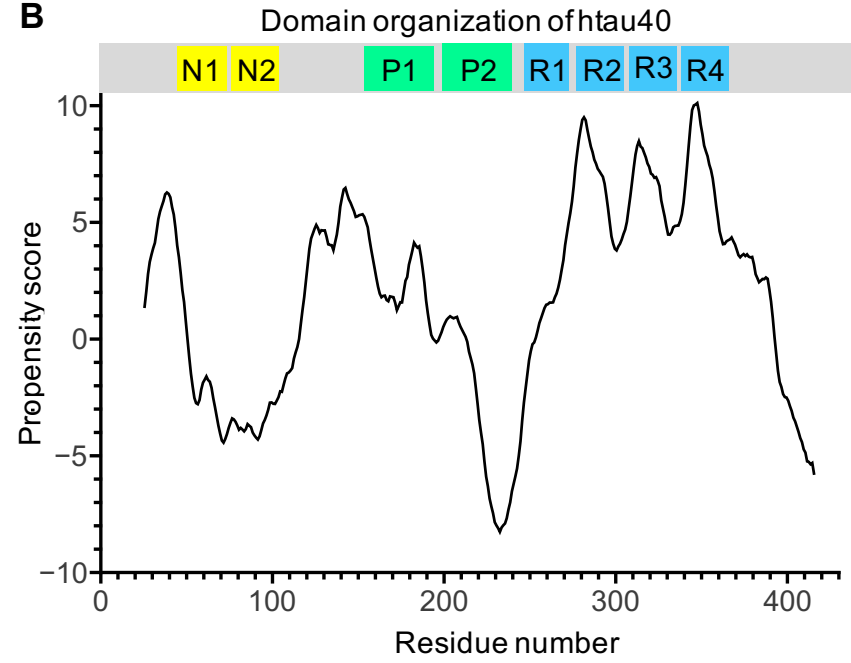

Figure 3. LLPS of tau protein. (A) Phase contrast and fluorescence micrographs of liquid-like droplets formed in a solution containing $50 \mu \mathrm{M}$ htau 40 in $25 \mathrm{mM}$ HEPES, $50 \mathrm{mM} \mathrm{KCl}, \mathrm{pH} 7.4$, and $10 \%$ of the molecular crowding agent dextran. Scale bars, $10 \mu \mathrm{m}$. (B) Residue-specific propensity scores for granule formation of htau 40 calculated by the software catGranule $[33,176]$. The domain organization of htau40 (2N4R tau; Fig. 2) is shown on top. 
Phosphorylation-dependent LLPS

tau concentration $=2 \mu \mathrm{M}, 10 \%(\mathrm{w} / \mathrm{v}) \mathrm{PEG}-8000,50 \mathrm{mM} \mathrm{NaCl}$
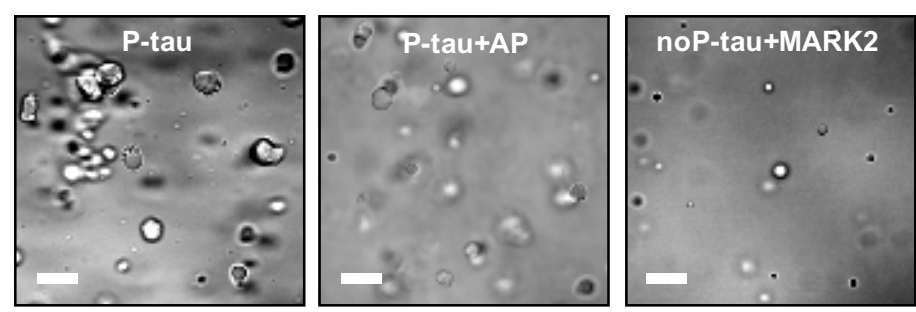

tau phosphorylation

tau LLPS rate, condensate size \& number, transition into aggregates

Figure 4. Tau phosphorylation influences tau condensation. Phosphorylated tau produced in Sf9 insect cells (P-tau), which carries an average of $\sim 12$ phosphates [147], undergoes LLPS upon addition of crowding agent (here $10 \% \mathrm{w} / \mathrm{v}$ polyethylene glycol (PEG-8000)) to a solution containing $2 \mu \mathrm{M}$ P-tau. De-phosphorylation of P-tau by alkaline phosphatase (P-tau+AP) reduces the formation of tau condensates and their size. In vitro phosphorylation of tau from E. coli (noP-tau) using the kinase MARK2 [106] enables tau LLPS at $2 \mu \mathrm{M}$ tau protein concentration [12,33]. LLPS of tau constructs was monitored by phase contrast microscopy 5.5 hours after addition of polyethylene glycol. See [12] for more details. Scale bars, $10 \mu \mathrm{m}$. 


\section{A}

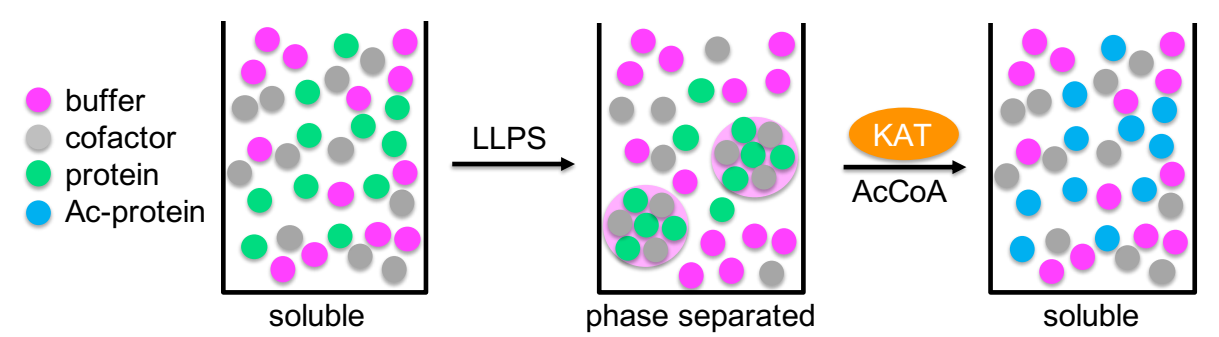

B

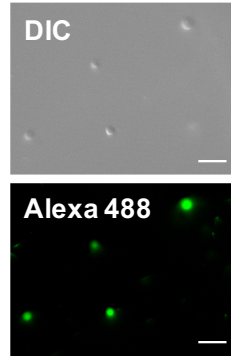

Acetylation dissolves htau40/dextran liquid droplets
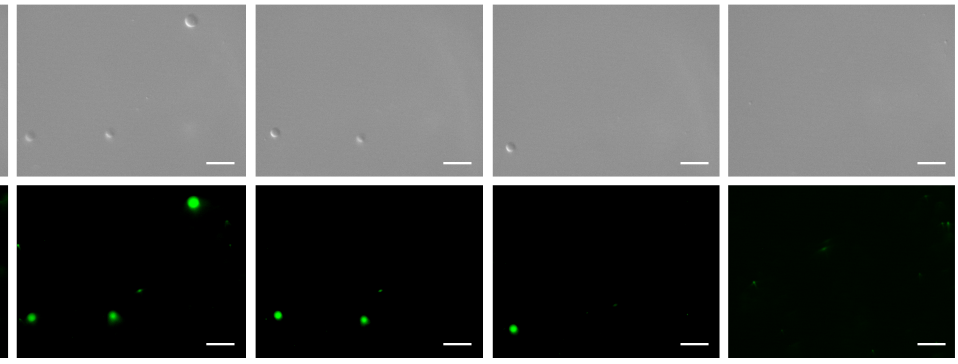

C

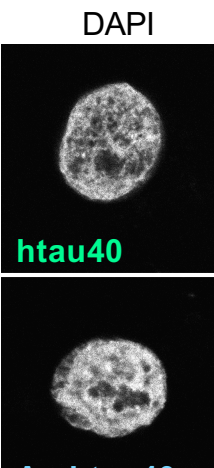

Alexa 488
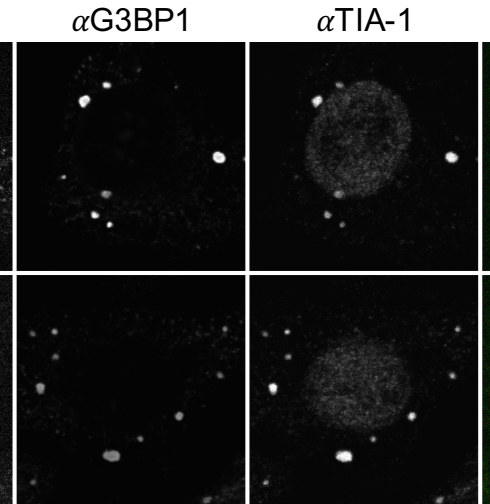

Merge
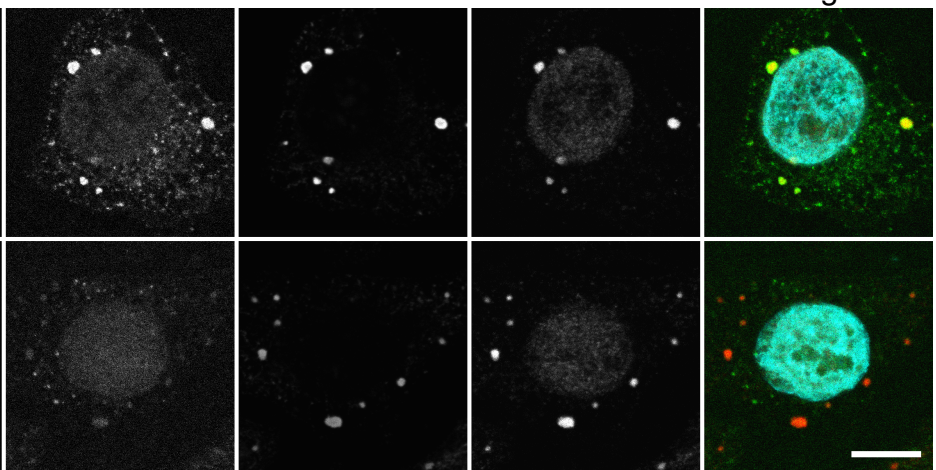

Figure 5. Acetylation dissolves tau droplets and attenuates colocalization of tau with stress granules [26]. (A) Cartoon representation illustrating tau liquid droplet formation in the presence of a cofactor and the influence of tau acetylation by lysine acetyltransferase (KAT) in presence of acetyl-Coenzyme A (AcCoA). (B) DIC and fluorescence images showing acetylation-induced dissolution of htau40/dextran (50 $\mu \mathrm{M}$ htau40, 10\% dextran in $25 \mathrm{mM}$ HEPES, $\mathrm{pH}$ 7.4) droplets in presence of CREB (KAT) and AcCoA. The images of the droplets were taken every 2 min $(2-10 \mathrm{~min})$. Scale bars, $10 \mu \mathrm{m}$. (C) After stress granules were first induced by proteasome inhibitor MG132 and the plasma membrane of Hela P4 cells was semi-permeabilized using digitonin, the cytosolic soluble factors were washed out and subsequently nuclear pore complexes were blocked by WGA. In the next step, Alexa 488-labeled htau40 and Ac-htau40 (acetylated htau40) were incubated with the semi-permeabilized cells to allow binding to SGs and after extensive washing, SGs were subjected to immunostaining for the SG makers G3BP1 and TIA-1. Scale bar, $10 \mu \mathrm{m}$. In the merge, Alexa 488 fluorescence and TIA-1 staining are shown in green and red, respectively. 
A

tauP301L expressing neurons
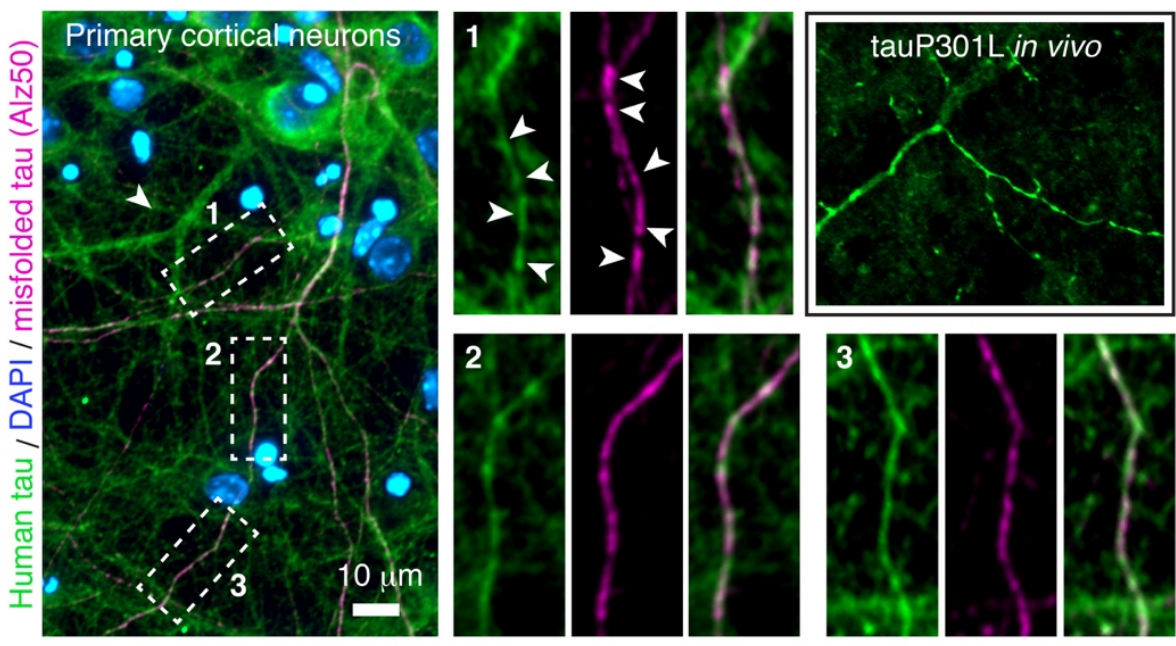

\section{B Cytosolic \\ GFP-tau condensate}

C Inhomogeneous distribution
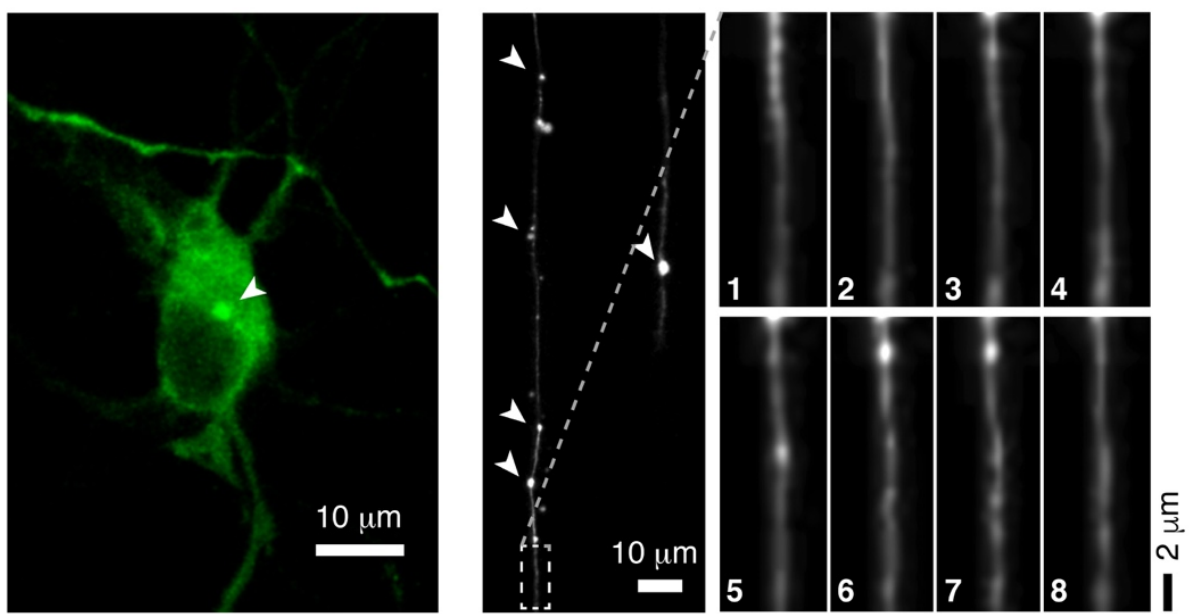

Figure 6. Liquid-like condensates and axonal "patches" of tau in neurons. (A) Primary cortical mouse neurons overexpressing human FTD-mutant tauP301L for seven days show an inhomogeneous distribution of human tau (green) in neuronal processes (zoom 1-3). The distribution of misfolded tau (magenta) appears even more "patchy" and is restricted to axons. Some of the axonal tau accumulations are indicated with white arrow heads. Neurons were transduced with an AAV encoding green fluorescent protein (GFP) and tauP301L (GFP-2a-tauP301L; [177]) on day in vitro (DIV) 7, then fixed and immunolabeled for human tau (TauY9 antibody) and misfolded tau (Alz50 antibody) on DIV14. Inhomogeneous distribution of tau in cortical neuronal processes can also be found in mice injected with the same AAV after 4 weeks. Sections from fixed brain were immunolabeled for human tau (Tau13 antibody). (B) Cortical mouse neurons show liquid-like GFP-tau condensates (white arrow head) and inhomogeneous distribution of GFP-tau in their processes already at $\sim 6-12 \mathrm{~h}$ of human GFP-tau expression in vitro. The example neuron was transfected with GFP-tau DNA at DIV7 and imaged $18 \mathrm{~h}$ after lipotransfection. (C) Fluorescence imaging of GFP-tau in axons of cortical neurons shows some bright droplet-like accumulations of GFP-tau along the axons (white arrow heads) and inhomogeneous and dynamic distribution of GFP-tau along axons (time-series images 1 to 8 of inset; one frame per 10 
seconds). To be able to unambiguously identify axonal compartments, primary mouse neurons were cultured in microfluidic chambers with long microchannels for axonal grow-through [178] for six days and then transfected with GFP-tau. Image and time series were taken 48-72 $\mathrm{h}$ after transfection with GFP-tau.

\section{References}

[1] S.F. Banani, H.O. Lee, A.A. Hyman, M.K. Rosen, Biomolecular condensates: organizers of cellular biochemistry, Nat Rev Mol Cell Biol 18 (5) (2017) 285-298.

[2] Y. Shin, C.P. Brangwynne, Liquid phase condensation in cell physiology and disease, Science 357 (6357) (2017).

[3] M. Feric, N. Vaidya, T.S. Harmon, D.M. Mitrea, L. Zhu, T.M. Richardson, R.W. Kriwacki, R.V. Pappu, C.P. Brangwynne, Coexisting Liquid Phases Underlie Nucleolar Subcompartments, Cell 165 (7) (2016) 1686-1697.

[4] T. Mittag, R. Parker, Multiple Modes of Protein-Protein Interactions Promote RNP Granule Assembly, J Mol Biol 430 (23) (2018) 4636-4649.

[5] A.C. Dumetz, A.M. Chockla, E.W. Kaler, A.M. Lenhoff, Effects of pH on protein-protein interactions and implications for protein phase behavior, Biochim Biophys Acta 1784 (4) (2008) 600-10.

[6] A.C. Dumetz, A.M. Chockla, E.W. Kaler, A.M. Lenhoff, Protein phase behavior in aqueous solutions: crystallization, liquid-liquid phase separation, gels, and aggregates, Biophys J 94 (2) (2008) 570-83.

[7] A.A. Hyman, C.A. Weber, F. Julicher, Liquid-liquid phase separation in biology, Annu Rev Cell Dev Biol 30 (2014) 39-58.

[8] A. Patel, H.O. Lee, L. Jawerth, S. Maharana, M. Jahnel, M.Y. Hein, S. Stoynov, J. Mahamid, S. Saha, T.M. Franzmann, A. Pozniakovski, I. Poser, N. Maghelli, L.A. Royer, M. Weigert, E.W. Myers, S. Grill, D. Drechsel, A.A. Hyman, S. Alberti, A Liquid-to-Solid Phase Transition of the ALS Protein FUS Accelerated by Disease Mutation, Cell 162 (5) (2015) 1066-77.

[9] A.E. Conicella, G.H. Zerze, J. Mittal, N.L. Fawzi, ALS Mutations Disrupt Phase Separation Mediated by alpha-Helical Structure in the TDP-43 Low-Complexity C-Terminal Domain, Structure 24 (9) (2016) 1537-49.

[10] P. St George-Hyslop, J.Q. Lin, A. Miyashita, E.C. Phillips, S. Qamar, S.J. Randle, G. Wang, The physiological and pathological biophysics of phase separation and gelation of RNA binding proteins in amyotrophic lateral sclerosis and fronto-temporal lobar degeneration, Brain Res 1693 (Pt A) (2018) 11-23.

[11] T. Murakami, S. Qamar, J.Q. Lin, G.S. Schierle, E. Rees, A. Miyashita, A.R. Costa, R.B. Dodd, F.T. Chan, C.H. Michel, D. Kronenberg-Versteeg, Y. Li, S.P. Yang, Y. Wakutani, W.

Meadows, R.R. Ferry, L. Dong, G.G. Tartaglia, G. Favrin, W.L. Lin, D.W. Dickson, M. Zhen, D. Ron, G. Schmitt-Ulms, P.E. Fraser, N.A. Shneider, C. Holt, M. Vendruscolo, C.F.

Kaminski, P. St George-Hyslop, ALS/FTD Mutation-Induced Phase Transition of FUS Liquid Droplets and Reversible Hydrogels into Irreversible Hydrogels Impairs RNP Granule Function, Neuron 88 (4) (2015) 678-90.

[12] S. Wegmann, B. Eftekharzadeh, K. Tepper, K.M. Zoltowska, R.E. Bennett, S. Dujardin, P.R. Laskowski, D. MacKenzie, T. Kamath, C. Commins, C. Vanderburg, A.D. Roe, Z. Fan, A.M. Molliex, A. Hernandez-Vega, D. Muller, A.A. Hyman, E. Mandelkow, J.P. Taylor, B.T. Hyman, Tau protein liquid-liquid phase separation can initiate tau aggregation, EMBO J 37 (7) (2018). 
[13] S. Qamar, G. Wang, S.J. Randle, F.S. Ruggeri, J.A. Varela, J.Q. Lin, E.C. Phillips, A. Miyashita, D. Williams, F. Strohl, W. Meadows, R. Ferry, V.J. Dardov, G.G. Tartaglia, L.A. Farrer, G.S. Kaminski Schierle, C.F. Kaminski, C.E. Holt, P.E. Fraser, G. Schmitt-Ulms, D. Klenerman, T. Knowles, M. Vendruscolo, P. St George-Hyslop, FUS Phase Separation Is Modulated by a Molecular Chaperone and Methylation of Arginine Cation-pi Interactions, Cell 173 (3) (2018) 720-734 e15.

[14] E. Bentmann, M. Neumann, S. Tahirovic, R. Rodde, D. Dormann, C. Haass, Requirements for stress granule recruitment of fused in sarcoma (FUS) and TAR DNAbinding protein of $43 \mathrm{kDa}$ (TDP-43), J Biol Chem 287 (27) (2012) 23079-94.

[15] J. Wang, J.M. Choi, A.S. Holehouse, H.O. Lee, X. Zhang, M. Jahnel, S. Maharana, R. Lemaitre, A. Pozniakovsky, D. Drechsel, I. Poser, R.V. Pappu, S. Alberti, A.A. Hyman, A Molecular Grammar Governing the Driving Forces for Phase Separation of Prion-like RNA Binding Proteins, Cell 174 (3) (2018) 688-699 e16.

[16] D.S.W. Protter, R. Parker, Principles and Properties of Stress Granules, Trends Cell Biol 26 (9) (2016) 668-679.

[17] P. Li, S. Banjade, H.C. Cheng, S. Kim, B. Chen, L. Guo, M. Llaguno, J.V.

Hollingsworth, D.S. King, S.F. Banani, P.S. Russo, Q.X. Jiang, B.T. Nixon, M.K. Rosen, Phase transitions in the assembly of multivalent signalling proteins, Nature 483 (7389) (2012) 336-40.

[18] W.M. Aumiller, Jr., C.D. Keating, Phosphorylation-mediated RNA/peptide complex coacervation as a model for intracellular liquid organelles, Nat Chem 8 (2) (2016) 129-37. [19] T.W. Han, M. Kato, S. Xie, L.C. Wu, H. Mirzaei, J. Pei, M. Chen, Y. Xie, J. Allen, G. Xiao, S.L. McKnight, Cell-free formation of RNA granules: bound RNAs identify features and components of cellular assemblies, Cell 149 (4) (2012) 768-79.

[20] Z. Monahan, V.H. Ryan, A.M. Janke, K.A. Burke, S.N. Rhoads, G.H. Zerze, R. O'Meally, G.L. Dignon, A.E. Conicella, W. Zheng, R.B. Best, R.N. Cole, J. Mittal, F.

Shewmaker, N.L. Fawzi, Phosphorylation of the FUS low-complexity domain disrupts phase separation, aggregation, and toxicity, EMBO J 36 (20) (2017) 2951-2967.

[21] T.J. Nott, E. Petsalaki, P. Farber, D. Jervis, E. Fussner, A. Plochowietz, T.D. Craggs,

D.P. Bazett-Jones, T. Pawson, J.D. Forman-Kay, A.J. Baldwin, Phase transition of a disordered nuage protein generates environmentally responsive membraneless organelles, Mol Cell 57 (5) (2015) 936-947.

[22] M. Hofweber, S. Hutten, B. Bourgeois, E. Spreitzer, A. Niedner-Boblenz, M. Schifferer, M.D. Ruepp, M. Simons, D. Niessing, T. Madl, D. Dormann, Phase Separation of FUS Is Suppressed by Its Nuclear Import Receptor and Arginine Methylation, Cell 173 (3) (2018) 706-719 e13.

[23] V.H. Ryan, G.L. Dignon, G.H. Zerze, C.V. Chabata, R. Silva, A.E. Conicella, J. Amaya, K.A. Burke, J. Mittal, N.L. Fawzi, Mechanistic View of hnRNPA2 Low-Complexity Domain Structure, Interactions, and Phase Separation Altered by Mutation and Arginine Methylation, Mol Cell 69 (3) (2018) 465-479 e7.

[24] J.C. Ferreon, A. Jain, K.J. Choi, P.S. Tsoi, K.R. MacKenzie, S.Y. Jung, A.C. Ferreon, Acetylation Disfavors Tau Phase Separation, Int J Mol Sci 19 (5) (2018).

[25] M. Saito, D. Hess, J. Eglinger, A.W. Fritsch, M. Kreysing, B.T. Weinert, C. Choudhary, P. Matthias, Acetylation of intrinsically disordered regions regulates phase separation, Nat Chem Biol 15 (1) (2019) 51-61.

[26] T. Ukmar-Godec, S. Hutten, M.P. Grieshop, N. Rezaei-Ghaleh, M.-S. Cima-Omori, J. Biernat, E. Mandelkow, J. Söding, D. Dormann, M. Zweckstetter, Lysine/RNA-interactions drive and regulate biomolecular condensation, Nat Commun (2019). DOI: 10.1038/s41467019-10792-y

[27] C.P. Brangwynne, P. Tompa, R.V. Pappu, Polymer physics of intracellular phase transitions, Nat Phys 11 (2015) 899-904. 
[28] D.R. Williams, Tauopathies: classification and clinical update on neurodegenerative diseases associated with microtubule-associated protein tau, Intern Med J 36 (10) (2006) 65260 .

[29] D.G. Drubin, M.W. Kirschner, Tau protein function in living cells, J Cell Biol 103 (6 Pt 2) (1986) 2739-46.

[30] V.M. Lee, B.J. Balin, L. Otvos, Jr., J.Q. Trojanowski, A68: a major subunit of paired helical filaments and derivatized forms of normal Tau, Science 251 (4994) (1991) 675-8. [31] C. Ballatore, V.M. Lee, J.Q. Trojanowski, Tau-mediated neurodegeneration in Alzheimer's disease and related disorders, Nat Rev Neurosci 8 (9) (2007) 663-72.

[32] L. Mucke, Neuroscience: Alzheimer's disease, Nature 461 (7266) (2009) 895-7.

[33] S. Ambadipudi, J. Biernat, D. Riedel, E. Mandelkow, M. Zweckstetter, Liquid-liquid phase separation of the microtubule-binding repeats of the Alzheimer-related protein Tau, Nat Commun 8 (1) (2017) 275.

[34] X. Zhang, Y. Lin, N.A. Eschmann, H. Zhou, J.N. Rauch, I. Hernandez, E. Guzman, K.S. Kosik, S. Han, RNA stores tau reversibly in complex coacervates, PLoS Biol 15 (7) (2017) e2002183.

[35] A. Hernandez-Vega, M. Braun, L. Scharrel, M. Jahnel, S. Wegmann, B.T. Hyman, S. Alberti, S. Diez, A.A. Hyman, Local Nucleation of Microtubule Bundles through Tubulin Concentration into a Condensed Tau Phase, Cell Rep 20 (10) (2017) 2304-2312.

[36] F. Comert, P.L. Dubin, Liquid-liquid and liquid-solid phase separation in proteinpolyelectrolyte systems, Adv Colloid Interface Sci 239 (2017) 213-217.

[37] H.G.B. de Jong, J. Bonner, Phosphatide auto-complex coacervates as ionic systems and their relation to the protoplasmic membrane, P K Akad Wet-Amsterd 38 (6/10) (1935) 797806.

[38] P.H. Poole, T. Grande, C.A. Angell, P.F. McMillan, Polymorphic phase transitions in liquids and glasses, Science 275 (5298) (1997) 322-323.

[39] P.J. Flory, Thermodynamics of High Polymer Solutions, J. Chem. Phys. 10 (1942) 5161.

[40] M.L. Huggins, Some Properties of Solutions of Long-chain Compounds, J. Chem. Phys. 46 (1) (1942) 151-158.

[41] J.T. Overbeek, M.J. Voorn, Phase separation in polyelectrolyte solutions; theory of complex coacervation, J Cell Physiol Suppl 49 (Suppl 1) (1957) 7-26.

[42] P.J. Flory, R.A. Orwoll, A. Vrij, Statistical Thermodynamics of Chain Molecule Liquids. I. An Equation of State for Normal Paraffin Hydrocarbons, J. Am. Chem. Soc. 86 (1964) 3507-3514.

[43] G. ten Brinke, F.E. Karasz, Lower Critical Solution Temperature Behavior in Polymer Blends: Compressibility and Directional-Specific Interactions, Macromolecules 17 (1984) 815-820.

[44] Y.H. Lin, J.D. Forman-Kay, H.S. Chan, Theories for Sequence-Dependent Phase Behaviors of Biomolecular Condensates, Biochemistry 57 (17) (2018) 2499-2508.

[45] A. Molliex, J. Temirov, J. Lee, M. Coughlin, A.P. Kanagaraj, H.J. Kim, T. Mittag, J.P. Taylor, Phase separation by low complexity domains promotes stress granule assembly and drives pathological fibrillization, Cell 163 (1) (2015) 123-33.

[46] H.R. Li, W.C. Chiang, P.C. Chou, W.J. Wang, J.R. Huang, TAR DNA-binding protein 43 (TDP-43) liquid-liquid phase separation is mediated by just a few aromatic residues, J Biol Chem 293 (16) (2018) 6090-6098.

[47] P.A. Chong, R.M. Vernon, J.D. Forman-Kay, RGG/RG Motif Regions in RNA Binding and Phase Separation, J Mol Biol 430 (23) (2018) 4650-4665.

[48] A.B. Kayitmazer, D. Seeman, B.B. Minsky, P.L. Dubin, Y.S. Xu, Proteinpolyelectrolyte interactions, Soft Matter 9 (2013) 2553-2583. 
[49] E.M. Langdon, Y. Qiu, A. Ghanbari Niaki, G.A. McLaughlin, C.A. Weidmann, T.M. Gerbich, J.A. Smith, J.M. Crutchley, C.M. Termini, K.M. Weeks, S. Myong, A.S. Gladfelter, mRNA structure determines specificity of a polyQ-driven phase separation, Science 360 (6391) (2018) 922-927.

[50] F.G. Quiroz, A. Chilkoti, Sequence heuristics to encode phase behaviour in intrinsically disordered protein polymers, Nat Mater 14 (11) (2015) 1164-71.

[51] H. Jiang, S. Wang, Y. Huang, X. He, H. Cui, X. Zhu, Y. Zheng, Phase transition of spindle-associated protein regulate spindle apparatus assembly, Cell 163 (1) (2015) 108-22.

[52] D.W. Urry, D.C. Gowda, T.M. Parker, C.H. Luan, M.C. Reid, C.M. Harris, A. Pattanaik, R.D. Harris, Hydrophobicity scale for proteins based on inverse temperature transitions, Biopolymers 32 (9) (1992) 1243-50.

[53] S.E. Reichheld, L.D. Muiznieks, F.W. Keeley, S. Sharpe, Direct observation of structure and dynamics during phase separation of an elastomeric protein, Proc Natl Acad Sci U S A 114 (22) (2017) E4408-E4415.

[54] R.J. Ellis, A.P. Minton, Cell biology: join the crowd, Nature 425 (6953) (2003) 27-8.

[55] M.L. Huggins, Theory of Solutions of High Polymers, J. Am. Chem. Soc. 64 (7) (1942) 1712-1719.

[56] A.P. Minton, Models for excluded volume interaction between an unfolded protein and rigid macromolecular cosolutes: macromolecular crowding and protein stability revisited, Biophys J 88 (2) (2005) 971-85.

[57] B.P. Paudel, E. Fiorini, R. Borner, R.K.O. Sigel, D.S. Rueda, Optimal molecular crowding accelerates group II intron folding and maximizes catalysis, Proc Natl Acad Sci U S A 115 (47) (2018) 11917-11922.

[58] S. Mukherjee, M.M. Waegele, P. Chowdhury, L. Guo, F. Gai, Effect of macromolecular crowding on protein folding dynamics at the secondary structure level, J Mol Biol 393 (1) (2009) 227-36.

[59] A. Soranno, I. Koenig, M.B. Borgia, H. Hofmann, F. Zosel, D. Nettels, B. Schuler, Single-molecule spectroscopy reveals polymer effects of disordered proteins in crowded environments, Proc Natl Acad Sci U S A 111 (13) (2014) 4874-9.

[60] A. Bhattacharya, Y.C. Kim, J. Mittal, Protein-protein interactions in a crowded environment, Biophys Rev 5 (2) (2013) 99-108.

[61] M. Sarkar, C. Li, G.J. Pielak, Soft interactions and crowding, Biophys Rev 5 (2) (2013) 187-194.

[62] M. Goedert, M.G. Spillantini, R. Jakes, D. Rutherford, R.A. Crowther, Multiple isoforms of human microtubule-associated protein tau: sequences and localization in neurofibrillary tangles of Alzheimer's disease, Neuron 3 (4) (1989) 519-26.

[63] M.D. Weingarten, A.H. Lockwood, S.Y. Hwo, M.W. Kirschner, A protein factor essential for microtubule assembly, Proc Natl Acad Sci U S A 72 (5) (1975) 1858-62.

[64] G. Lee, N. Cowan, M. Kirschner, The primary structure and heterogeneity of tau protein from mouse brain, Science 239 (4837) (1988) 285-8.

[65] N. Gustke, B. Trinczek, J. Biernat, E.M. Mandelkow, E. Mandelkow, Domains of tau protein and interactions with microtubules, Biochemistry 33 (32) (1994) 9511-22.

[66] K.A. Butner, M.W. Kirschner, Tau protein binds to microtubules through a flexible array of distributed weak sites, J Cell Biol 115 (3) (1991) 717-30.

[67] M.D. Mukrasch, S. Bibow, J. Korukottu, S. Jeganathan, J. Biernat, C. Griesinger, E. Mandelkow, M. Zweckstetter, Structural polymorphism of 441-residue tau at single residue resolution, PLoS Biol 7 (2) (2009) e34.

[68] M.D. Mukrasch, M. von Bergen, J. Biernat, D. Fischer, C. Griesinger, E. Mandelkow, M. Zweckstetter, The "jaws" of the tau-microtubule interaction, J Biol Chem 282 (16) (2007) 12230-9. 
[69] H. Kadavath, M. Jaremko, L. Jaremko, J. Biernat, E. Mandelkow, M. Zweckstetter, Folding of the Tau Protein on Microtubules, Angew Chem Int Ed Engl 54 (35) (2015) 1034751 .

[70] H. Kadavath, R.V. Hofele, J. Biernat, S. Kumar, K. Tepper, H. Urlaub, E. Mandelkow, M. Zweckstetter, Tau stabilizes microtubules by binding at the interface between tubulin heterodimers, Proc Natl Acad Sci U S A 112 (24) (2015) 7501-6.

[71] E.H. Kellogg, N.M.A. Hejab, S. Poepsel, K.H. Downing, F. DiMaio, E. Nogales, Nearatomic model of microtubule-tau interactions, Science 360 (6394) (2018) 1242-1246.

[72] D.W. Cleveland, S.Y. Hwo, M.W. Kirschner, Physical and chemical properties of purified tau factor and the role of tau in microtubule assembly, J Mol Biol 116 (2) (1977) 22747.

[73] H.J. Dyson, P.E. Wright, Intrinsically unstructured proteins and their functions, Nat Rev Mol Cell Biol 6 (3) (2005) 197-208.

[74] N. Rezaei-Ghaleh, M. Blackledge, M. Zweckstetter, Intrinsically disordered proteins: from sequence and conformational properties toward drug discovery, Chembiochem 13 (7) (2012) 930-50.

[75] T. Lee, C.R. Moran-Gutierrez, A.A. Deniz, Probing protein disorder and complexity at single-molecule resolution, Semin Cell Dev Biol 37 (2015) 26-34.

[76] S. Elbaum-Garfinkle, E. Rhoades, Identification of an aggregation-prone structure of tau, J Am Chem Soc 134 (40) (2012) 16607-13.

[77] A.M. Melo, J. Coraor, G. Alpha-Cobb, S. Elbaum-Garfinkle, A. Nath, E. Rhoades, A functional role for intrinsic disorder in the tau-tubulin complex, Proc Natl Acad Sci U S A 113 (50) (2016) 14336-14341.

[78] B. Schuler, A. Soranno, H. Hofmann, D. Nettels, Single-Molecule FRET Spectroscopy and the Polymer Physics of Unfolded and Intrinsically Disordered Proteins, Annu Rev Biophys 45 (2016) 207-31.

[79] A. Nath, M. Sammalkorpi, D.C. DeWitt, A.J. Trexler, S. Elbaum-Garfinkle, C.S. O'Hern, E. Rhoades, The conformational ensembles of alpha-synuclein and tau: combining singlemolecule FRET and simulations, Biophys J 103 (9) (2012) 1940-9.

[80] M. von Bergen, P. Friedhoff, J. Biernat, J. Heberle, E.M. Mandelkow, E. Mandelkow, Assembly of tau protein into Alzheimer paired helical filaments depends on a local sequence motif ((306)VQIVYK(311)) forming beta structure, Proc Natl Acad Sci U S A 97 (10) (2000) 5129-34.

[81] O. Schweers, E.M. Mandelkow, J. Biernat, E. Mandelkow, Oxidation of cysteine-322 in the repeat domain of microtubule-associated protein tau controls the in vitro assembly of paired helical filaments, Proc Natl Acad Sci U S A 92 (18) (1995) 8463-7.

[82] Z.Y. Mo, Y.Z. Zhu, H.L. Zhu, J.B. Fan, J. Chen, Y. Liang, Low micromolar zinc accelerates the fibrillization of human tau via bridging of Cys-291 and Cys-322, J Biol Chem 284 (50) (2009) 34648-57.

[83] M.D. Mukrasch, J. Biernat, M. von Bergen, C. Griesinger, E. Mandelkow, M. Zweckstetter, Sites of tau important for aggregation populate \{beta\}-structure and bind to microtubules and polyanions, J Biol Chem 280 (26) (2005) 24978-86.

[84] S. Barghorn, P. Davies, E. Mandelkow, Tau paired helical filaments from Alzheimer's disease brain and assembled in vitro are based on beta-structure in the core domain, Biochemistry 43 (6) (2004) 1694-703.

[85] M. von Bergen, S. Barghorn, J. Biernat, E.M. Mandelkow, E. Mandelkow, Tau aggregation is driven by a transition from random coil to beta sheet structure, Biochim Biophys Acta 1739 (2-3) (2005) 158-66.

[86] J. Berriman, L.C. Serpell, K.A. Oberg, A.L. Fink, M. Goedert, R.A. Crowther, Tau filaments from human brain and from in vitro assembly of recombinant protein show crossbeta structure, Proc Natl Acad Sci U S A 100 (15) (2003) 9034-8. 
[87] V. Daebel, S. Chinnathambi, J. Biernat, M. Schwalbe, B. Habenstein, A. Loquet, E. Akoury, K. Tepper, H. Muller, M. Baldus, C. Griesinger, M. Zweckstetter, E. Mandelkow, V. Vijayan, A. Lange, beta-Sheet core of tau paired helical filaments revealed by solid-state NMR, J Am Chem Soc 134 (34) (2012) 13982-9.

[88] A.W.P. Fitzpatrick, B. Falcon, S. He, A.G. Murzin, G. Murshudov, H.J. Garringer, R.A. Crowther, B. Ghetti, M. Goedert, S.H.W. Scheres, Cryo-EM structures of tau filaments from Alzheimer's disease, Nature 547 (7662) (2017) 185-190.

[89] B. Falcon, J. Zivanov, W. Zhang, A.G. Murzin, H.J. Garringer, R. Vidal, R.A. Crowther, K.L. Newell, B. Ghetti, M. Goedert, S.H.W. Scheres, Novel tau filament fold in chronic traumatic encephalopathy encloses hydrophobic molecules, Nature 568 (7752) (2019) 420423.

[90] S. Bibow, M.D. Mukrasch, S. Chinnathambi, J. Biernat, C. Griesinger, E. Mandelkow, M. Zweckstetter, The dynamic structure of filamentous tau, Angew Chem Int Ed Engl 50 (48) (2011) 11520-4.

[91] G.A. Jicha, R. Bowser, I.G. Kazam, P. Davies, Alz-50 and MC-1, a new monoclonal antibody raised to paired helical filaments, recognize conformational epitopes on recombinant tau, J Neurosci Res 48 (2) (1997) 128-32.

[92] G. Carmel, E.M. Mager, L.I. Binder, J. Kuret, The structural basis of monoclonal antibody Alz50's selectivity for Alzheimer's disease pathology, J Biol Chem 271 (51) (1996) 32789-95.

[93] E. Prezel, A. Elie, J. Delaroche, V. Stoppin-Mellet, C. Bosc, L. Serre, A. FourestLieuvin, A. Andrieux, M. Vantard, I. Arnal, Tau can switch microtubule network organizations: from random networks to dynamic and stable bundles, Mol Biol Cell 29 (2) (2018) 154-165.

[94] P. Giannakopoulos, F.R. Herrmann, T. Bussiere, C. Bouras, E. Kovari, D.P. Perl, J.H. Morrison, G. Gold, P.R. Hof, Tangle and neuron numbers, but not amyloid load, predict cognitive status in Alzheimer's disease, Neurology 60 (9) (2003) 1495-500.

[95] C. Bancher, H. Braak, P. Fischer, K.A. Jellinger, Neuropathological staging of Alzheimer lesions and intellectual status in Alzheimer's and Parkinson's disease patients, Neurosci Lett 162 (1-2) (1993) 179-82.

[96] D.A. Olszewska, R. Lonergan, E.M. Fallon, T. Lynch, Genetics of Frontotemporal Dementia, Curr Neurol Neurosci Rep 16 (12) (2016) 107.

[97] M. von Bergen, S. Barghorn, L. Li, A. Marx, J. Biernat, E.M. Mandelkow, E. Mandelkow, Mutations of tau protein in frontotemporal dementia promote aggregation of paired helical filaments by enhancing local beta-structure, J Biol Chem 276 (51) (2001) 48165-74.

[98] H. Wille, G. Drewes, J. Biernat, E.M. Mandelkow, E. Mandelkow, Alzheimer-like paired helical filaments and antiparallel dimers formed from microtubule-associated protein tau in vitro, J Cell Biol 118 (3) (1992) 573-84.

[99] M. Goedert, R. Jakes, M.G. Spillantini, M. Hasegawa, M.J. Smith, R.A. Crowther, Assembly of microtubule-associated protein tau into Alzheimer-like filaments induced by sulphated glycosaminoglycans, Nature 383 (6600) (1996) 550-3.

[100] P. Friedhoff, M. von Bergen, E.M. Mandelkow, P. Davies, E. Mandelkow, A nucleated assembly mechanism of Alzheimer paired helical filaments, Proc Natl Acad Sci U S A 95 (26) (1998) 15712-7.

[101] T.C. Gamblin, F. Chen, A. Zambrano, A. Abraha, S. Lagalwar, A.L. Guillozet, M. Lu, Y. Fu, F. Garcia-Sierra, N. LaPointe, R. Miller, R.W. Berry, L.I. Binder, V.L. Cryns, Caspase cleavage of tau: linking amyloid and neurofibrillary tangles in Alzheimer's disease, Proc Natl Acad Sci U S A 100 (17) (2003) 10032-7.

[102] Z. Zhang, M. Song, X. Liu, S.S. Kang, I.S. Kwon, D.M. Duong, N.T. Seyfried, W.T. Hu, Z. Liu, J.Z. Wang, L. Cheng, Y.E. Sun, S.P. Yu, A.I. Levey, K. Ye, Cleavage of tau by 
asparagine endopeptidase mediates the neurofibrillary pathology in Alzheimer's disease, Nat Med 20 (11) (2014) 1254-62.

[103] M. Morris, G.M. Knudsen, S. Maeda, J.C. Trinidad, A. Ioanoviciu, A.L. Burlingame, L. Mucke, Tau post-translational modifications in wild-type and human amyloid precursor protein transgenic mice, Nat Neurosci 18 (8) (2015) 1183-9.

[104] L. Martin, X. Latypova, F. Terro, Post-translational modifications of tau protein: implications for Alzheimer's disease, Neurochem Int 58 (4) (2011) 458-71.

[105] M. Schwalbe, H. Kadavath, J. Biernat, V. Ozenne, M. Blackledge, E. Mandelkow, M. Zweckstetter, Structural Impact of Tau Phosphorylation at Threonine 231, Structure 23 (8) (2015) 1448-1458.

[106] M. Schwalbe, J. Biernat, S. Bibow, V. Ozenne, M.R. Jensen, H. Kadavath, M. Blackledge, E. Mandelkow, M. Zweckstetter, Phosphorylation of human Tau protein by microtubule affinity-regulating kinase 2, Biochemistry 52 (50) (2013) 9068-79.

[107] S. Bibow, V. Ozenne, J. Biernat, M. Blackledge, E. Mandelkow, M. Zweckstetter, Structural impact of proline-directed pseudophosphorylation at AT8, AT100, and PHF1 epitopes on 441-residue tau, J Am Chem Soc 133 (40) (2011) 15842-5.

[108] D. Fischer, M.D. Mukrasch, J. Biernat, S. Bibow, M. Blackledge, C. Griesinger, E. Mandelkow, M. Zweckstetter, Conformational changes specific for pseudophosphorylation at serine 262 selectively impair binding of tau to microtubules, Biochemistry 48 (42) (2009) 10047-55.

[109] Y. Cabrales Fontela, H. Kadavath, J. Biernat, D. Riedel, E. Mandelkow, M.

Zweckstetter, Multivalent cross-linking of actin filaments and microtubules through the microtubule-associated protein Tau, Nat Commun 8 (1) (2017) 1981.

[110] H. Zempel, E. Mandelkow, Lost after translation: missorting of Tau protein and consequences for Alzheimer disease, Trends Neurosci 37 (12) (2014) 721-32.

[111] C. Li, J. Gotz, Tau-based therapies in neurodegeneration: opportunities and challenges, Nat Rev Drug Discov 16 (12) (2017) 863-883.

[112] C.X. Gong, F. Liu, I. Grundke-Iqbal, K. Iqbal, Post-translational modifications of tau protein in Alzheimer's disease, J Neural Transm (Vienna) 112 (6) (2005) 813-38.

[113] S.W. Min, S.H. Cho, Y. Zhou, S. Schroeder, V. Haroutunian, W.W. Seeley, E.J. Huang, Y. Shen, E. Masliah, C. Mukherjee, D. Meyers, P.A. Cole, M. Ott, L. Gan, Acetylation of tau inhibits its degradation and contributes to tauopathy, Neuron 67 (6) (2010) 953-66.

[114] T.J. Cohen, J.L. Guo, D.E. Hurtado, L.K. Kwong, I.P. Mills, J.Q. Trojanowski, V.M. Lee, The acetylation of tau inhibits its function and promotes pathological tau aggregation, Nat Commun 2 (2011) 252.

[115] T.E. Tracy, P.D. Sohn, S.S. Minami, C. Wang, S.W. Min, Y. Li, Y. Zhou, D. Le, I. Lo, R. Ponnusamy, X. Cong, B. Schilling, L.M. Ellerby, R.L. Huganir, L. Gan, Acetylated Tau Obstructs KIBRA-Mediated Signaling in Synaptic Plasticity and Promotes Tauopathy-Related Memory Loss, Neuron 90 (2) (2016) 245-60.

[116] B. Kuhla, C. Haase, K. Flach, H.J. Luth, T. Arendt, G. Munch, Effect of pseudophosphorylation and cross-linking by lipid peroxidation and advanced glycation end product precursors on tau aggregation and filament formation, J Biol Chem 282 (10) (2007) 6984-91.

[117] M.D. Ledesma, M. Medina, J. Avila, The in vitro formation of recombinant tau polymers: effect of phosphorylation and glycation, Mol Chem Neuropathol 27 (3) (1996) 24958.

[118] M.A. Smith, M. Tabaton, G. Perry, Early contribution of oxidative glycation in Alzheimer disease, Neurosci Lett 217 (2-3) (1996) 210-1.

[119] M. Necula, J. Kuret, Pseudophosphorylation and glycation of tau protein enhance but do not trigger fibrillization in vitro, J Biol Chem 279 (48) (2004) 49694-703. 
[120] M. Takahashi, Y. Tsujioka, T. Yamada, Y. Tsuboi, H. Okada, T. Yamamoto, Z. Liposits, Glycosylation of microtubule-associated protein tau in Alzheimer's disease brain, Acta Neuropathol 97 (6) (1999) 635-41.

[121] J.Z. Wang, I. Grundke-Iqbal, K. Iqbal, Glycosylation of microtubule-associated protein tau: an abnormal posttranslational modification in Alzheimer's disease, Nat Med 2 (8) (1996) $871-5$.

[122] L.A. Robertson, K.L. Moya, K.C. Breen, The potential role of tau protein Oglycosylation in Alzheimer's disease, J Alzheimers Dis 6 (5) (2004) 489-95.

[123] T. Lefebvre, S. Ferreira, L. Dupont-Wallois, T. Bussiere, M.J. Dupire, A. Delacourte, J.C. Michalski, M.L. Caillet-Boudin, Evidence of a balance between phosphorylation and OGlcNAc glycosylation of Tau proteins--a role in nuclear localization, Biochim Biophys Acta 1619 (2) (2003) 167-76.

[124] F. Liu, K. Iqbal, I. Grundke-Iqbal, C.X. Gong, Involvement of aberrant glycosylation in phosphorylation of tau by cdk5 and GSK-3beta, FEBS Lett 530 (1-3) (2002) 209-14.

[125] G. Drewes, A. Ebneth, U. Preuss, E.M. Mandelkow, E. Mandelkow, MARK, a novel family of protein kinases that phosphorylate microtubule-associated proteins and trigger microtubule disruption, Cell 89 (2) (1997) 297-308.

[126] S. Ambadipudi, J.G. Reddy, J. Biernat, E. Mandelkow, M. Zweckstetter, Residuespecific identification of liquid phase separation hot spots of the Alzheimer's disease-related protein Tau, Chem Sci (2019). DOI: 10.1039/C9SC00531E

[127] S. Konzack, E. Thies, A. Marx, E.M. Mandelkow, E. Mandelkow, Swimming against the tide: mobility of the microtubule-associated protein tau in neurons, J Neurosci 27 (37) (2007) 9916-27.

[128] T. Kampers, P. Friedhoff, J. Biernat, E.M. Mandelkow, E. Mandelkow, RNA stimulates aggregation of microtubule-associated protein tau into Alzheimer-like paired helical filaments, FEBS Lett 399 (3) (1996) 344-9.

[129] X. Wang, D. Wang, J. Zhao, M. Qu, X. Zhou, H. He, R. He, The proline-rich domain and the microtubule binding domain of protein tau acting as RNA binding domains, Protein Pept Lett 13 (7) (2006) 679-85.

[130] J. Lefevre, K.G. Chernov, V. Joshi, S. Delga, F. Toma, D. Pastre, P.A. Curmi, P. Savarin, The $\mathrm{C}$ terminus of tubulin, a versatile partner for cationic molecules: binding of Tau, polyamines, and calcium, J Biol Chem 286 (4) (2011) 3065-78.

[131] M.G. Spillantini, M. Goedert, Tau mutations in familial frontotemporal dementia, Brain 123 ( Pt 5) (2000) 857-9.

[132] S. Boyko, X. Qi, T.H. Chen, K. Surewicz, W.K. Surewicz, Liquid-liquid phase separation of tau protein: The crucial role of electrostatic interactions, J Biol Chem (2019).

[133] T. Vanderweyde, H. Yu, M. Varnum, L. Liu-Yesucevitz, A. Citro, T. Ikezu, K. Duff, B. Wolozin, Contrasting pathology of the stress granule proteins TIA-1 and G3BP in tauopathies, J Neurosci 32 (24) (2012) 8270-83.

[134] P.A. Chong, J.D. Forman-Kay, Liquid-liquid phase separation in cellular signaling systems, Curr Opin Struct Biol 41 (2016) 180-186.

[135] C.P. Brangwynne, C.R. Eckmann, D.S. Courson, A. Rybarska, C. Hoege, J.

Gharakhani, F. Julicher, A.A. Hyman, Germline P granules are liquid droplets that localize by controlled dissolution/condensation, Science 324 (5935) (2009) 1729-32.

[136] M. Kato, T.W. Han, S. Xie, K. Shi, X. Du, L.C. Wu, H. Mirzaei, E.J. Goldsmith, J.

Longgood, J. Pei, N.V. Grishin, D.E. Frantz, J.W. Schneider, S. Chen, L. Li, M.R. Sawaya, D. Eisenberg, R. Tycko, S.L. McKnight, Cell-free formation of RNA granules: low complexity sequence domains form dynamic fibers within hydrogels, Cell 149 (4) (2012) 753-67. [137] S.F. Banani, A.M. Rice, W.B. Peeples, Y. Lin, S. Jain, R. Parker, M.K. Rosen, Compositional Control of Phase-Separated Cellular Bodies, Cell 166 (3) (2016) 651-663. 
[138] Y. Lin, J. McCarty, J.N. Rauch, K.T. Delaney, K.S. Kosik, G.H. Fredrickson, J.-E. Shea, S. Han, Narrow equilibrium window for complex coacervation of tau and RNA under cellular conditions, Elife 8 (2019) pii: e42571.

[139] A.L. Darling, V.N. Uversky, Intrinsic Disorder and Posttranslational Modifications: The Darker Side of the Biological Dark Matter, Front Genet 9 (2018) 158.

[140] A. Bah, J.D. Forman-Kay, Modulation of Intrinsically Disordered Protein Function by Post-translational Modifications, J Biol Chem 291 (13) (2016) 6696-705.

[141] C. Bancher, C. Brunner, H. Lassmann, H. Budka, K. Jellinger, F. Seitelberger, I. Grundke-Iqbal, K. Iqbal, H.M. Wisniewski, Tau and ubiquitin immunoreactivity at different stages of formation of Alzheimer neurofibrillary tangles, Prog Clin Biol Res 317 (1989) 83748 .

[142] E. Kopke, Y.C. Tung, S. Shaikh, A.C. Alonso, K. Iqbal, I. Grundke-Iqbal, Microtubuleassociated protein tau. Abnormal phosphorylation of a non-paired helical filament pool in Alzheimer disease, J Biol Chem 268 (32) (1993) 24374-84.

[143] C.X. Gong, K. Iqbal, Hyperphosphorylation of microtubule-associated protein tau: a promising therapeutic target for Alzheimer disease, Curr Med Chem 15 (23) (2008) 2321-8. [144] H. Braak, E. Braak, Morphological criteria for the recognition of Alzheimer's disease and the distribution pattern of cortical changes related to this disorder, Neurobiol Aging 15 (3) (1994) 355-6; discussion 379-80.

[145] G. Drewes, B. Trinczek, S. Illenberger, J. Biernat, G. Schmitt-Ulms, H.E. Meyer, E.M. Mandelkow, E. Mandelkow, Microtubule-associated protein/microtubule affinity-regulating kinase (p110mark). A novel protein kinase that regulates tau-microtubule interactions and dynamic instability by phosphorylation at the Alzheimer-specific site serine 262, J Biol Chem 270 (13) (1995) 7679-88.

[146] J. Biernat, N. Gustke, G. Drewes, E.M. Mandelkow, E. Mandelkow, Phosphorylation of Ser262 strongly reduces binding of tau to microtubules: distinction between PHF-like immunoreactivity and microtubule binding, Neuron 11 (1) (1993) 153-63.

[147] K. Tepper, J. Biernat, S. Kumar, S. Wegmann, T. Timm, S. Hubschmann, L. Redecke, E.M. Mandelkow, D.J. Muller, E. Mandelkow, Oligomer formation of tau protein hyperphosphorylated in cells, J Biol Chem 289 (49) (2014) 34389-407.

[148] X.J. Yang, E. Seto, Lysine acetylation: codified crosstalk with other posttranslational modifications, Mol Cell 31 (4) (2008) 449-61.

[149] B.M. Dancy, P.A. Cole, Protein lysine acetylation by p300/CBP, Chem Rev 115 (6) (2015) 2419-52.

[150] G. Breuzard, P. Hubert, R. Nouar, T. De Bessa, F. Devred, P. Barbier, J.N. Sturgis, V. Peyrot, Molecular mechanisms of Tau binding to microtubules and its role in microtubule dynamics in live cells, J Cell Sci 126 (Pt 13) (2013) 2810-9.

[151] R. Dixit, J.L. Ross, Y.E. Goldman, E.L. Holzbaur, Differential regulation of dynein and kinesin motor proteins by tau, Science 319 (5866) (2008) 1086-9.

[152] D.P. McVicker, G.J. Hoeprich, A.R. Thompson, C.L. Berger, Tau interconverts between diffusive and stable populations on the microtubule surface in an isoform and lattice specific manner, Cytoskeleton (Hoboken) 71 (3) (2014) 184-94.

[153] A. Samsonov, J.Z. Yu, M. Rasenick, S.V. Popov, Tau interaction with microtubules in vivo, J Cell Sci 117 (Pt 25) (2004) 6129-41.

[154] R. Brandt, G. Lee, The balance between tau protein's microtubule growth and nucleation activities: implications for the formation of axonal microtubules, J Neurochem 61 (3) (1993) 997-1005.

[155] H. Felgner, R. Frank, M. Schliwa, Flexural rigidity of microtubules measured with the use of optical tweezers, J Cell Sci 109 ( Pt 2) (1996) 509-16. 
[156] F. Breitling, M. Little, Carboxy-terminal regions on the surface of tubulin and microtubules. Epitope locations of YOL1/34, DM1A and DM1B, J Mol Biol 189 (2) (1986) 367-70.

[157] U.Z. Littauer, D. Giveon, M. Thierauf, I. Ginzburg, H. Ponstingl, Common and distinct tubulin binding sites for microtubule-associated proteins, Proc Natl Acad Sci U S A 83 (19) (1986) 7162-6.

[158] H.P. Erickson, W.A. Voter, Polycation-induced assembly of purified tubulin, Proc Natl Acad Sci U S A 73 (8) (1976) 2813-7.

[159] V. Siahaan, J. Krattenmacher, A. Hernandez-Vega, A.A. Hyman, A.A. Hyman, Z. Lansky, M. Braun, Kinetically distinct phases of tau on microtubules regulate kinesin motors and severing enzymes, BioRxiv 424374 (2018).

[160] R. Tan, A.J. Lam, T. Tan, J. Han, D.W. Nowakowski, M. Vershinin, S. Simo, K.M. OriMcKenney, R.J. McKenney, Microtubules Gate Tau Condensation to Spatially Regulate Microtubule Functions, BioRxiv 423376 (2018).

[161] M.H. Hinrichs, A. Jalal, B. Brenner, E. Mandelkow, S. Kumar, T. Scholz, Tau protein diffuses along the microtubule lattice, J Biol Chem 287 (46) (2012) 38559-68.

[162] D. Janning, M. Igaev, F. Sundermann, J. Bruhmann, O. Beutel, J.J. Heinisch, L. Bakota, J. Piehler, W. Junge, R. Brandt, Single-molecule tracking of tau reveals fast kiss-and-hop interaction with microtubules in living neurons, Mol Biol Cell 25 (22) (2014) 3541-51.

[163] L. Qiang, X. Sun, T.O. Austin, H. Muralidharan, D.C. Jean, M. Liu, W. Yu, P.W. Baas, Tau Does Not Stabilize Axonal Microtubules but Rather Enables Them to Have Long Labile Domains, Curr Biol 28 (13) (2018) 2181-2189 e4.

[164] F.J. Dennissen, M. Anglada-Huguet, A. Sydow, E. Mandelkow, E.M. Mandelkow, Adenosine A1 receptor antagonist rolofylline alleviates axonopathy caused by human Tau DeltaK280, Proc Natl Acad Sci U S A 113 (41) (2016) 11597-11602.

[165] A. Ebneth, R. Godemann, K. Stamer, S. Illenberger, B. Trinczek, E. Mandelkow, Overexpression of tau protein inhibits kinesin-dependent trafficking of vesicles, mitochondria, and endoplasmic reticulum: implications for Alzheimer's disease, J Cell Biol 143 (3) (1998) 777-94.

[166] K. Stamer, R. Vogel, E. Thies, E. Mandelkow, E.M. Mandelkow, Tau blocks traffic of organelles, neurofilaments, and APP vesicles in neurons and enhances oxidative stress, J Cell Biol 156 (6) (2002) 1051-63.

[167] F. Paonessa, L. Evans, R. Solanki, D. Larrieu, S. Wray, J. Hardy, S.P. Jackson, F.J. Livesey, Microtubules Deform the Nuclear Membrane and Disrupt Nucleocytoplasmic Transport in Tau-Mediated Frontotemporal Dementia, Cell Rep 26 (3) (2019) 582-593.e5. [168] K. Moschner, F. Sundermann, H. Meyer, A.P. da Graca, N. Appel, A. Paululat, L. Bakota, R. Brandt, RNA protein granules modulate tau isoform expression and induce neuronal sprouting, J Biol Chem 289 (24) (2014) 16814-25.

[169] S. Aronov, G. Aranda, L. Behar, I. Ginzburg, Axonal tau mRNA localization coincides with tau protein in living neuronal cells and depends on axonal targeting signal, J Neurosci 21 (17) (2001) 6577-87.

[170] S. Aronov, G. Aranda, L. Behar, I. Ginzburg, Visualization of translated tau protein in the axons of neuronal P19 cells and characterization of tau RNP granules, J Cell Sci 115 (Pt 19) (2002) 3817-27.

[171] A. Aulas, M.M. Fay, S.M. Lyons, C.A. Achorn, N. Kedersha, P. Anderson, P. Ivanov, Stress-specific differences in assembly and composition of stress granules and related foci, $\mathrm{J}$ Cell Sci 130 (5) (2017) 927-937.

[172] T. Vanderweyde, D.J. Apicco, K. Youmans-Kidder, P.E.A. Ash, C. Cook, E. Lummertz da Rocha, K. Jansen-West, A.A. Frame, A. Citro, J.D. Leszyk, P. Ivanov, J.F. Abisambra, M. Steffen, H. Li, L. Petrucelli, B. Wolozin, Interaction of tau with the RNA-Binding Protein TIA1 Regulates tau Pathophysiology and Toxicity, Cell Rep 15 (7) (2016) 1455-1466. 
[173] D.J. Apicco, P.E.A. Ash, B. Maziuk, C. LeBlang, M. Medalla, A. Al Abdullatif, A. Ferragud, E. Botelho, H.I. Ballance, U. Dhawan, S. Boudeau, A.L. Cruz, D. Kashy, A. Wong, L.R. Goldberg, N. Yazdani, C. Zhang, C.Y. Ung, Y. Tripodis, N.M. Kanaan, T. Ikezu, P. Cottone, J. Leszyk, H. Li, J. Luebke, C.D. Bryant, B. Wolozin, Reducing the RNA binding protein TIA1 protects against tau-mediated neurodegeneration in vivo, Nat Neurosci 21 (1) (2018) 72-80.

[174] B.F. Maziuk, D.J. Apicco, A.L. Cruz, L. Jiang, P.E.A. Ash, E.L. da Rocha, C. Zhang, W.H. Yu, J. Leszyk, J.F. Abisambra, H. Li, B. Wolozin, RNA binding proteins co-localize with small tau inclusions in tauopathy, Acta Neuropathol Commun 6 (1) (2018) 71.

[175] S. Boeynaems, E. Bogaert, D. Kovacs, A. Konijnenberg, E. Timmerman, A. Volkov, M. Guharoy, M. De Decker, T. Jaspers, V.H. Ryan, A.M. Janke, P. Baatsen, T. Vercruysse, R.M. Kolaitis, D. Daelemans, J.P. Taylor, N. Kedersha, P. Anderson, F. Impens, F. Sobott, J.

Schymkowitz, F. Rousseau, N.L. Fawzi, W. Robberecht, P. Van Damme, P. Tompa, L. Van Den Bosch, Phase Separation of C9orf72 Dipeptide Repeats Perturbs Stress Granule Dynamics, Mol Cell 65 (6) (2017) 1044-1055 e5.

[176] B. Bolognesi, N. Lorenzo Gotor, R. Dhar, D. Cirillo, M. Baldrighi, G.G. Tartaglia, B. Lehner, A Concentration-Dependent Liquid Phase Separation Can Cause Toxicity upon Increased Protein Expression, Cell Rep 16 (1) (2016) 222-231.

[177] S. Wegmann, R.E. Bennett, A.S. Amaral, B.T. Hyman, Studying tau protein propagation and pathology in the mouse brain using adeno-associated viruses, Methods Cell Biol 141 (2017) 307-322.

[178] S. Takeda, S. Wegmann, H. Cho, S.L. DeVos, C. Commins, A.D. Roe, S.B. Nicholls, G.A. Carlson, R. Pitstick, C.K. Nobuhara, I. Costantino, M.P. Frosch, D.J. Muller, D. Irimia, B.T. Hyman, Neuronal uptake and propagation of a rare phosphorylated high-molecularweight tau derived from Alzheimer's disease brain, Nat Commun 6 (2015) 8490. 